\title{
THE VANISHING CONJECTURE FOR MAPS OF TOR AND DERIVED SPLINTERS
}

\author{
LINQUAN MA
}

\begin{abstract}
We say an excellent local domain $(S, \mathfrak{n})$ satisfies the vanishing conditions for maps of Tor, if for every $A \rightarrow R \rightarrow S$ with $A$ regular and $A \rightarrow R$ module-finite torsionfree extension, and every $A$-module $M$, the map $\operatorname{Tor}_{i}^{A}(M, R) \rightarrow \operatorname{Tor}_{i}^{A}(M, S)$ vanishes for every $i \geq 1$. Hochster-Huneke's conjecture (theorem in equal characteristic) thus states that regular rings satisfy such vanishing conditions [HH95. The main theorem of this paper shows that, in equal characteristic, rings that satisfy the vanishing conditions for maps of Tor are exactly derived splinters in the sense of Bhatt [Bha12]. In particular, rational singularities in characteristic 0 satisfy the vanishing conditions. This greatly generalizes Hochster-Huneke's result [HH95] and Boutot's theorem Bou87. Moreover, our result leads to a new (and surprising) characterization of rational singularities in terms of splittings in module-finite extensions.
\end{abstract}

\section{INTRODUCTION}

In [HH95, Hochster and Huneke proved the following extremely strong vanishing result in equal characteristic:

Theorem 1.1 ( $c f$. Theorem 4.1 in [HH95]). Let $A$ be an equal characteristic regular domain, let $R$ be a module-finite and torsion-free extension of $A$, and let $R \rightarrow S$ be any homomorphism from $R$ to a regular ring $S$. Then for every $A$-module $M$ and every $i \geq 1$, the map $\operatorname{Tor}_{i}^{A}(M, R) \rightarrow \operatorname{Tor}_{i}^{A}(M, S)$ vanishes.

It is also conjectured by Hochster and Huneke that Theorem 1.1 holds in mixed characteristic. This is one of the well-known homological conjectures: the vanishing conjecture for maps of Tor. The importance of Theorem 1.1, as well as the corresponding conjecture in mixed characteristic, lies in the fact that, in any characteristic, it implies both the direct summand conjecture and the conjecture that direct summands of regular rings are CohenMacaulay [HH95]. Indeed, it was shown in Ran00] that the vanishing conjecture for maps of Tor is equivalent to a strong form of the direct summand conjecture (we refer to Ran00] for details).

In fact, results very similar to Theorem 1.1 were first proved in [HH93], in characteristic $p>0$ only, using tight closure and phantom homology theory 1 The proof given in [HH95] makes crucial use of the existence of weakly functorial balanced big Cohen-Macaulay algebras in equal characteristic. In characteristic $p>0$, the existence of such algebras follows directly from [HH92], where it was shown that the absolute integral closure $R^{+}$is such an algebra. In

\footnotetext{
${ }^{1}$ It is pointed out in the introduction of HH93 that by reduction to characteristic $p>0$, one can develop the corresponding theory in characteristic 0 . The full results in HH93 are, in some sense, even stronger than Theorem 1.1 but are slightly technical to state here. However we point out that all these (stronger) results can be established by the argument used in [HH95. Our method can provide generalizations of these results also, both in characteristic $p>0$ and characteristic 0 , see Remark [5.6.
} 
characteristic 0 , the construction of weakly functorial balanced big Cohen-Macaulay algebras depends on a very delicate and difficult reduction to characteristic $p>0$ argument (we refer to Section 3 of [HH95] for details). In mixed characteristic, the analogy of Theorem 1.1 is known when $A, R, S$ all have dimension less than or equal to three [Hoc02, based on Heitmann's results Hei02]. However, in general the vanishing conjecture for maps of Tor is wide open in mixed characteristic.

In this paper, we investigate Theorem 1.1 in some new and different ways. We study the "converse" of Theorem 1.1 in the following sense: in a given characteristic, for which local domain $S$, the map $\operatorname{Tor}_{i}^{A}(M, R) \rightarrow \operatorname{Tor}_{i}^{A}(M, S)$ vanishes for every $A \rightarrow R \rightarrow S$ and every $A$-module $M$ (where $A$ is regular and $A \rightarrow R$ is a module-finite torsion-free extension)? We will say such $S$ satisfies the vanishing conditions for maps of Tor (see Section 2 for precise definitions). We will show that, in all characteristics, such vanishing conditions imply $S$ has only pseudo-rational singularities, which is a characteristic-free analogue of rational singularities. Our main result in equal characteristic is the following:

Theorem 1.2 (=Theorem 5.5) . Let $S$ be a local domain that is essentially of finite type over a field. The following are equivalent:

(1) S satisfies the vanishing conditions for maps of Tor.

(2) $S$ is a derived splinter.

(3) For every regular local ring $A$ with $S=A / P$ and every module-finite torsion-free extension $A \rightarrow B$ with $Q \in \operatorname{Spec} B$ lying over $P, P \rightarrow Q$ splits as $A$-modules.

We note that derived splinters are formally introduced by Bhatt in [Bha12], and are well understood in equal characteristic: they are equivalent to rational singularities in characteristic 0 [Kov00], Bha12] and in characteristic $p>0$, they turn out to be the same as splinters [Bha12] (see Section 2 for precise definitions for splinters and derived splinters). In fact, at least in characteristic 0, the idea of derived splinters plays a crucial role in our proofs.

As regular local rings in equal characteristic are derived splinters, Theorem $1.2(1) \Leftrightarrow(2)$ greatly extends Theorem 1.1. We will see in Remark 5.7 that Theorem 1.2 also generalizes Boutot's theorem that direct summands of rational singularities are rational singularities [Bou87 (Boutot's theorem follows from the vanishing of Tor applied to $M=E_{A}$, the injective hull of $A$ ). Moreover, as an immediate consequence of Theorem 1.2 (2) $\Leftrightarrow(3)$, we obtain the following new characterization of rational singularities. We find this characterization surprising as it only addresses splittings in module-finite extensions.

Corollary 1.3 (=Corollary [5.8). Let $(S, \mathfrak{n})$ be a local domain essentially of finite type over a field of characteristic 0 . Then $S$ has rational singularities if and only if for every regular local ring $A$ with $S=A / P$, every module-finite torsion-free extension $A \rightarrow T$, and every $Q \in \operatorname{Spec} T$ lying over $P$, the map $P \rightarrow Q$ splits as a map of $A$-modules.

This paper is organized as follows. In Section 2, we recall and review the basic theories, and we introduce two important concepts: the vanishing conditions for maps of Tor and the vanishing conditions for maps of local cohomology. The rest of the paper is devoted to the proof of Theorem 1.2. In Section 3 we show that the vanishing conditions for maps of Tor implies pseudo-rationality. In Section 4 we prove $(1) \Leftrightarrow(3)$ in Theorem 1.2. Finally, in Section 5 we prove $(1) \Leftrightarrow(2)$ in Theorem 1.2 and we also prove some partial results in mixed characteristic: for example, we show that the vanishing conjecture for maps of Tor implies the derived direct summand conjecture. Throughout this paper, unless otherwise stated, we 
will make the following assumptions on commutative rings and schemes (we will sometimes repeat and emphasize these conditions):

(1) All rings are Noetherian, excellent and are homomorphic image of regular rings.

(2) All schemes are Noetherian, separated, excellent that admit dualizing complexes.

(3) In characteristic 0, all rings and schemes are essentially of finite type over a field.

We point out that (1) and (2) are very mild conditions (e.g., all rings essentially of finite type over a complete local ring satisfy (1)). We make the assumption (3) mainly because we need to apply the Grauert-Riemenschneider type vanishing theorems [GR70], [Kol86] in characteristic 0.

\section{Definitions and Preliminaries}

We begin with some basic definitions of plus closure. Let $S$ be an integral domain and $I \subseteq S$ be an ideal. The plus closure of $I, I^{+}$, is the set of elements $x \in S$ such that $x \in I T$ for some module-finite extension $T$ of $S . I$ is called plus closed if $I^{+}=I$. The absolute integral closure of $S$, denoted by $S^{+}$, is the integral closure of $S$ in the algebraic closure of the fraction field of $S$, which is also the direct limit of all the module-finite domain extensions of $S$ [HH92]. It follows that $I^{+}=I S^{+} \bigcap S$. The plus closure of 0 in $H_{\mathfrak{n}}^{d}(S)$, the top local cohomology module, is defined as $0_{H_{\mathfrak{n}}^{d}(S)}^{+}=\operatorname{ker}\left(H_{\mathfrak{n}}^{d}(S) \rightarrow H_{\mathfrak{n}}^{d}\left(S^{+}\right)\right)$.

A domain $S$ (resp., An integral scheme $X$ ) is called a splinter, if for every module-finite extension $T$ of $S$ (resp., every finite surjective map $Y \rightarrow X$ ), the natural map $S \rightarrow T$ (resp., $O_{X} \rightarrow O_{Y}$ ) is split in the category of $S$-modules (resp., $O_{X}$-modules). It is easy to see that $S$ is a splinter if and only if every ideal in $S$ is plus closed.

Let $(S, \mathfrak{n})$ be an excellent local domain of characteristic $p>0$. The top local cohomology module $H_{\mathfrak{n}}^{d}(S)$ has a natural Frobenius action. In this situation, there is a unique largest proper submodule of $H_{\mathfrak{n}}^{d}(S)$ that is stable under the Frobenius action, which is $0_{H_{\mathfrak{n}}^{d}(S)}^{*}$, the tight closure of 0 in $H_{\mathfrak{n}}^{d}(S)$ [Smi97]. $(S, \mathfrak{n})$ is called $F$-rational, if it is Cohen-Macaulay and $0_{H_{\mathrm{n}}^{d}(S)}^{*}=0$ [HH94], Smi97]. This is not the original definition of F-rationality, but it turns out to be extremely useful in many applications. It is worth mentioning that a deep result of Smith [Smi94] shows that $0_{H_{\mathfrak{n}}^{d}(S)}^{*}=0_{H_{\mathfrak{n}}^{d}(S)}^{+}$, which we will need in Section 5.

We make some more comments on splinters. In equal characteristic 0 , using the trace map, it is straightforward to check that splinters are exactly normal schemes. However, even in equal characteristic $p>0$ in the affine case, splinters are quite mysterious. It is known that affine splinters in characteristic $p>0$ are always $F$-rational [Smi94] Bha12, and it is conjectured that they are $F$-regular, which is a natural strengthening of $F$-rationality and an important concept in tight closure theory 2 We refer to [Sin99] and [CEMS14] for the best partial results on this conjecture. In mixed characteristic, our knowledge about splinters is minimal: Hochster's famous direct summand conjecture asserts that regular local rings are splinters. This conjecture is known in dimension $\leq 3$ [Hei02], and is open (in mixed characteristic) in dimension $\geq 4$.

Following [Bha12, we say an integral scheme $X$ is a derived splinter, if for any proper surjective map $f: Y \rightarrow X$, the pullback map $O_{X} \rightarrow \mathbf{R} f_{*} O_{Y}$ is split in the derived category $D(\operatorname{Coh}(X))$ of coherent sheaves on $X$. This is the same as requiring $O_{X} \rightarrow \mathbf{R} f_{*} O_{Y}$ to split

${ }^{2}$ As we will not use deep results in tight closure theory, we omit the precise definition of $F$-regularity (and the original definition of $F$-rationality). We refer to [HH90] for details on tight closure theory. 
in $D(\mathrm{QCoh}(X))$, the derived category of quasi-coherent sheaves on $X$. It is easy to see that derived splinters are splinters. It was first observed in [Kov00 that derived splinters in characteristic 0 coincide with rational singularities, 3 while it was shown in Bha12] that, quite surprisingly, derived splinters are equivalent to splinters in characteristic $p>0$.

Next we recall pseudo-rational singularities [LT81]: A $d$-dimensional local $\operatorname{ring}(R, \mathfrak{m})$ is called pseudo-rational if it is normal, Cohen-Macaulay, analytically unramified (i.e., the completion $\widehat{R}$ is reduced), and if for every proper, birational map $\pi$ : $W \rightarrow \operatorname{Spec} R$ with $W$ normal, the canonical map $H_{\mathfrak{m}}^{d}(R) \rightarrow H_{E}^{d}\left(W, O_{W}\right)$ is injective where $E=\pi^{-1}(\mathfrak{m})$ denotes the closed fibre. Pseudo-rationality is a property of local rings which is an analog of rational singularities for more general schemes, e.g., rings which may not have a desingularization. When the ring is essentially of finite type over a field of characteristic 0, pseudo-rational singularities are the same as rational singularities. In characteristic $p$, pseudo-rationality is slightly weaker than F-rationality [Smi97], [Har98].

We summarize the relations between these concepts. In characteristic 0, we have:

$$
\text { derived } \text { splinter }=\text { rational singularities }=\text { pseudo-rational } \Longrightarrow \text { splinter }
$$

In characteristic $p>0$, we have:

$$
\text { derived splinter }=\text { splinter } \Longrightarrow F \text {-rational } \Longrightarrow \text { pseudo-rational. }
$$

Now we introduce the central concepts that we will study in this paper:

Definition 2.1. We say a local domain $(S, \mathfrak{n})$ satisfies the vanishing conditions for maps of Tor, if for every $A \rightarrow R \rightarrow S$ such that $A$ is a regular domain, $A \rightarrow R$ is a modulefinite torsion-free extension, and $A, R, S$ have the same characteristic 4 the natural map $\operatorname{Tor}_{i}^{A}(M, R) \rightarrow \operatorname{Tor}_{i}^{A}(M, S)$ vanishes for every $A$-module $M$ and every $i \geq 1$.

It is also quite natural to ask that: if $(R, \mathfrak{m}) \rightarrow(S, \mathfrak{n})$ is a surjection of local domains, when does $H_{\mathfrak{m}}^{j}(R) \rightarrow H_{\mathfrak{n}}^{j}(S)$ vanish for every $j<\operatorname{dim} R$ ? (this is inspired by Corollary 4.24 of [HH93], which itself is a consequence of Theorem 1.1). Hence similar to the vanishing conditions for maps of Tor, we want to introduce certain vanishing conditions for maps of local cohomology. Since there are several equivalent ways to define this, we summarize them into a proposition.

Proposition/Definition 2.2. Let $(S, \mathfrak{n})$ be a local domain of dimension $d$. Then the following are equivalent (we always assume $R, S$ have the same characteristic):

(1) For every surjection $(R, \mathfrak{m}) \rightarrow(S, \mathfrak{n})$ with $(R, \mathfrak{m})$ equidimensional, the induced map $H_{\mathfrak{m}}^{j}(R) \rightarrow H_{\mathfrak{n}}^{j}(S)$ vanishes for every $j<\operatorname{dim} R$.

(2) For every surjection $(R, \mathfrak{m}) \rightarrow(S, \mathfrak{n})$ with $(R, \mathfrak{m})$ a local domain, the induced map $H_{\mathfrak{m}}^{j}(R) \rightarrow H_{\mathfrak{n}}^{j}(S)$ vanishes for every $j<\operatorname{dim} R$.

(3) $S$ is Cohen-Macaulay and for every surjection $(R, \mathfrak{m}) \rightarrow(S, \mathfrak{n})$ such that $(R, \mathfrak{m})$ is a local domain with $\operatorname{dim} R>d$, the induced map $H_{\mathfrak{m}}^{d}(R) \rightarrow H_{\mathfrak{n}}^{d}(S)$ vanishes.

(4) $S$ is Cohen-Macaulay and for every surjection $(R, \mathfrak{m}) \rightarrow(S, \mathfrak{n})$ such that $\operatorname{dim} R / P>d$ for every minimal prime of $P$ of $R$, the induced map $H_{\mathfrak{m}}^{d}(R) \rightarrow H_{\mathfrak{n}}^{d}(S)$ vanishes.

${ }^{3}$ This was proved in Kov00 when $Y \rightarrow X$ has connected fibres (which was suffices for the applications in Kov00). A complete proof was given in Theorem 2.12 in Bha12.

${ }^{4}$ This means $A, R, S$ all have equal characteristic, i.e., they all contain a field, or they all have mixed characteristic (i.e., the characteristic of the ring is different from that of its residue field). 
We say $(S, \mathfrak{n})$ satisfies the vanishing conditions for maps of local cohomology, if it satisfies the above equivalent conditions.

Proof. (1) $\Rightarrow(2)$ : This is obvious.

$(2) \Rightarrow(3)$ : Applying (2) to $R=S$, we get that the identity map $H_{\mathfrak{n}}^{j}(S) \rightarrow H_{\mathfrak{n}}^{j}(S)$ vanishes for every $j<\operatorname{dim} S=d$. Thus $S$ is Cohen-Macaulay. The remaining part is obvious (note that one cannot apply (3) to $R=S$, because the hypothesis on $R$ in (3) forces $\operatorname{dim} R>d$ ).

$(3) \Rightarrow(4)$ : Since $S$ is a domain, every surjection $R \rightarrow S$ factors through $R \rightarrow R^{\prime} \rightarrow S$, where $R^{\prime}=R / P$ for some minimal prime $P$ of $R$. Now (3) implies $H_{\mathfrak{m}}^{d}\left(R^{\prime}\right) \rightarrow H_{\mathfrak{n}}^{d}(S)$ vanishes because $\operatorname{dim} R^{\prime}=\operatorname{dim} R / P>d$. Thus $H_{\mathfrak{m}}^{d}(R) \rightarrow H_{\mathfrak{n}}^{d}(S)$ also vanishes.

(4) $\Rightarrow$ (1): If $\operatorname{dim} R=\operatorname{dim} S=d$ (i.e., $R=S$ ) in (1), then $H_{\mathfrak{m}}^{j}(R) \rightarrow H_{\mathfrak{n}}^{j}(S)$ vanishes for every $j<\operatorname{dim} R=d$ because $H_{\mathfrak{n}}^{j}(S)=0$ ( $S$ is Cohen-Macaulay). Otherwise we have $\operatorname{dim} R>d$. Since $R$ is equidimensional, $\operatorname{dim} R / P>d$ for every minimal prime $P$ of $R$. Thus applying (4), we know that $H_{\mathfrak{m}}^{d}(R) \rightarrow H_{\mathfrak{n}}^{d}(S)$ vanishes.

Remark 2.3. One cannot expect that $H_{\mathfrak{m}}^{d}(R) \rightarrow H_{\mathfrak{n}}^{d}(S)$ vanish for all $R \rightarrow S$ with $\operatorname{dim} R>d$, even when $S$ is regular. For example, let $R=\frac{k[[x, y, z]]}{(x, y) \cap(z)}$ and $S=k[[z]]$. We know that $\operatorname{dim} R=2$ and $\operatorname{dim} S=1$. But it is easy to check that $H_{\mathfrak{m}}^{1}(R) \rightarrow H_{\mathfrak{n}}^{1}(S)$ is surjective and hence does not vanish. The trouble here is that there is a component of $R$ that has the same dimension as $S$. Thus the hypotheses in Definition $2.2(1)-(4)$ are necessary.

We will see in later sections that the vanishing conditions for Tor and for local cohomology are deeply related: Proposition 3.4 , Theorem 5.10.

\section{VANishing OF TOR, VANISHING OF LOCAL COHOMOLOGY AND PSEUdO-RATIONALITY}

In this section we will show that the vanishing conditions for maps of Tor implies pseudorationality, which will be a crucial ingredient in proving $(1) \Rightarrow(2)$ in Theorem 1.2 . We also obtain many characteristic-free results of independent interest.

Lemma 3.1. Let $(S, \mathfrak{n})$ be a local domain that is a homomorphic image of a regular ring. Then we have:

$$
\sum_{R} \operatorname{im}\left(H_{\mathfrak{m}}^{d}(R) \rightarrow H_{\mathfrak{n}}^{d}(S)\right) \supseteq 0_{H_{\mathfrak{n}}^{d}(S)}^{+}
$$

where the sum is taken over all $R \rightarrow S$ such that $\operatorname{dim} R / P>\operatorname{dim} S=d$ for every minimal prime $P$ of $R$. In particular, if $(S, \mathfrak{n})$ satisfies the vanishing conditions for maps of local cohomology, then we have $0_{H_{\mathfrak{n}}^{d}(S)}^{+}=0$.

Proof. Since $0_{H_{\mathfrak{n}}^{d}(S)}^{+}=\operatorname{ker}\left(H_{\mathfrak{n}}^{d}(S) \rightarrow H_{\mathfrak{n}}^{d}\left(S^{+}\right)\right)=\cup_{T} \operatorname{ker}\left(H_{\mathfrak{n}}^{d}(S) \rightarrow H_{\mathfrak{n}}^{d}(T)\right)$ where $T$ runs over all module-finite domain extensions of $S$. It suffices to show that $\sum_{R} \operatorname{im}\left(H_{\mathfrak{m}}^{d}(R) \rightarrow H_{\mathfrak{n}}^{d}(S)\right) \supseteq$ $\operatorname{ker}\left(H_{\mathfrak{n}}^{d}(S) \rightarrow H_{\mathfrak{n}}^{d}(T)\right)$ for every such $T$.

We write $S=A / P$ for some regular local $\operatorname{ring} A$ such that $\operatorname{dim} A \geq d+1$. Let $t_{1}, \ldots, t_{n}$ be a set of generators of $T$ over $S$. Since $T$ is integral over $S$, each $t_{i}$ satisfies a monic

polynomial $f_{i}$ over $S$. We can lift each $f_{i}$ to $A$ and form the ring $B=\frac{A\left[x_{1}, \ldots, x_{n}\right]}{\left(f_{1}, \ldots, f_{n}\right)}$. We have a natural surjective map $B \rightarrow T$ with kernel $Q \in \operatorname{Spec} B$. It is clear that $Q$ lies over $P$ in $A$. 
Let $R=A+Q \subseteq B$. We know that $R / Q=A / P=S$. In sum, we have:

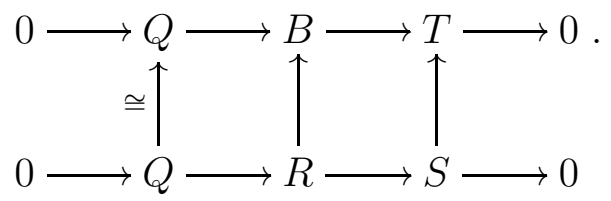

Let $\mathfrak{m}$ be the pre-image of $\mathfrak{n}$ in $R$. Because $B$ is free over $A$ and $R$ is a subring of $B, R$ is torsion-free over $A$. Now localizing at $\mathfrak{m}$ if necessary, we know that $(R, \mathfrak{m})$ is equidimensional and $\operatorname{dim} R=\operatorname{dim} B=\operatorname{dim} A \geq d+1$. This guarantees that $\operatorname{dim} R / P>d$ for every minimal prime $P$ of $R$. The induced long exact sequences on local cohomology gives:

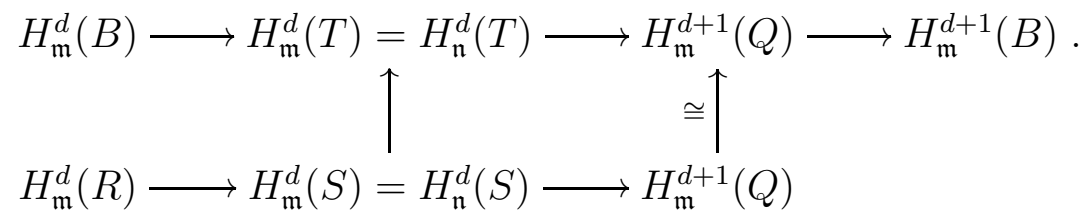

Chasing this diagram, it is easy to see that:

$$
\operatorname{ker}\left(H_{\mathfrak{n}}^{d}(S) \rightarrow H_{\mathfrak{n}}^{d}(T)\right) \subseteq \operatorname{im}\left(H_{\mathfrak{m}}^{d}(R) \rightarrow H_{\mathfrak{n}}^{d}(S)\right) .
$$

This proves (3.1.1). Finally, if $S$ satisfies the vanishing conditions for maps of local cohomology, then the left hand side of (3.1.1) is 0 by Definition $2.2(4)$, thus $0_{H_{\mathfrak{n}}^{d}(S)}^{+}=0$.

Corollary 3.2. If $(S, \mathfrak{n})$ satisfies the vanishing conditions for maps of local cohomology, then every ideal generated by a full system of parameters in $S$ is plus closed. In particular this implies $S$ is normal.

Proof. Let $I=\left(x_{1}, \ldots, x_{d}\right)$ be any ideal generated by a full system of parameters of $S$. Consider the commutative diagram:

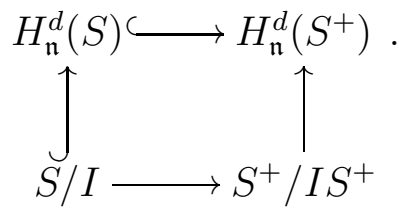

The left vertical map is injective because $S$ is Cohen-Macaulay by Definition 2.2, and the map in the top row is injective by Lemma 3.1. Chasing this diagram we know that $S / I \hookrightarrow S^{+} / I S^{+}$ is injective. This proves that $I$ is plus closed.

Finally, every ideal generated by a system of parameters is plus closed implies that every ideal generated by part of a system of parameters is plus closed: suppose $\left(x_{1}, \ldots, x_{t}\right)$ is part of a system of parameters, contained in $\left(x_{1}, \ldots, x_{t}, x_{t+1}, \ldots, x_{d}\right)$. If $y \in\left(x_{1}, \ldots, x_{t}\right)^{+}$, then $y \in\left(x_{1}, \ldots, x_{t}, x_{t+1}^{s}, \ldots, x_{d}^{s}\right)^{+}=\left(x_{1}, \ldots, x_{t}, x_{t+1}^{s}, \ldots, x_{d}^{s}\right)$ for every $s>0$. So

$$
y \in \bigcap_{s}\left(x_{1}, \ldots, x_{t}, x_{t+1}^{s}, \ldots, x_{d}^{s}\right)=\left(x_{1}, \ldots, x_{t}\right) \text {. }
$$

In particular, we know that every principal ideal is plus closed. Let $y \in \overline{(x)}$, the integral closure of the ideal generated by $x$. Then $y \in \overline{(x) R^{+}}=(x) R^{+}$because $R^{+}$is integrally closed. So $y \in(x)^{+}=(x)$. This proves every principal ideal is integrally closed and hence $S$ is normal. 
Lemma 3.3. If $S$ satisfies the vanishing conditions for maps of local cohomology, then $S$ is pseudo-rational.

Proof. By our general assumption on commutative rings, $S$ is an excellent local domain hence is analytically unramified. By Definition 2.2 and Corollary 3.2, $S$ is Cohen-Macaulay and normal. To check the last condition of pseudo-rationality, we let $W \rightarrow \operatorname{Spec} S$ be a proper birational map with $W$ normal, and we can assume this map is projective and birational by Chow's Lemma. Therefore $W \rightarrow$ Spec $S$ is just the blow up of some ideal $J$ in $S$, i.e.,

$$
W=\operatorname{Proj} S \oplus J t \oplus J^{2} t^{2} \oplus \cdots:=\operatorname{Proj} R .
$$

Now we apply the Sancho de Salas exact sequence (see page 202 of [SdS87], or take cohomology of (5.11.1) in Section 5) to $W=\operatorname{Proj} R \rightarrow \operatorname{Spec} S$ to get $(d=\operatorname{dim} S)$ :

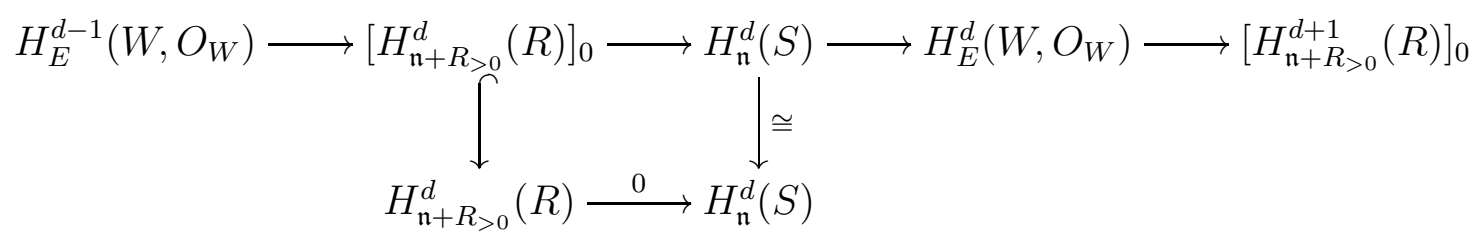

Since $R$ has dimension $d+1$ and $S$ satisfies the vanishing conditions for maps of local cohomology, the bottom map is the zero map. By the commutativity of the above diagram, we see the map $\left[H_{\mathfrak{n}+R_{>0}}^{d}(R)\right]_{0} \rightarrow H_{\mathfrak{n}}^{d}(S)$ vanishes. Therefore $H_{\mathfrak{n}}^{d}(S) \rightarrow H_{E}^{d}\left(W, O_{W}\right)$ is injective. This finishes the proof.

Proposition 3.4. When $S$ is a complete local domain, $S$ satisfies the vanishing conditions for maps of Tor implies $S$ satisfies the vanishing conditions for maps of local cohomology. Hence both implies $S$ has only pseudo-rational singularities.

Proof. Let $(R, \mathfrak{m}) \rightarrow(S, \mathfrak{n})$ be a surjection with $R$ a domain. We may complete $R$ to get $\widehat{R} \rightarrow \widehat{S}=S$. Since $R$ is excellent by our general assumptions on commutative rings, $\widehat{R}$ is equidimensional. Since $S$ is a domain, the map $\widehat{R} \rightarrow S$ factors through $\widehat{R} \rightarrow R^{\prime} \rightarrow S$ where $R^{\prime}=\widehat{R} / P$ for $P$ a minimal prime of $\widehat{R}$. Thus in order to show $H_{\mathfrak{m}}^{j}(R) \rightarrow H_{\mathfrak{n}}^{j}(S)$ vanishes for $j<\operatorname{dim} R$, it suffices to show $H_{\mathfrak{m}}^{j}\left(R^{\prime}\right) \rightarrow H_{\mathfrak{n}}^{j}(S)$ vanishes for $j<\operatorname{dim} R^{\prime}$. Hence without loss of generality, we may replace $R$ by $R^{\prime}$ and assume $R$ is a complete local domain.

Now by Cohen's structure theorem, we have $\left(A, \mathfrak{m}_{0}\right) \hookrightarrow(R, \mathfrak{m})$ a module-finite extension with $\left(A, \mathfrak{m}_{0}\right)$ regular local. Let $E_{A}=E_{A}\left(A / \mathfrak{m}_{0}\right) \cong H_{\mathfrak{m}_{0}}^{n}(A)$ be the injective hull of the residue field of $A$. Since the Cech complex gives a flat resolution of $E_{A}$, we know that $\operatorname{Tor}_{i}^{A}\left(E_{A}, R\right) \cong$ $H_{\mathfrak{m}}^{n-i}(R)$ and $\operatorname{Tor}_{i}^{A}\left(E_{A}, S\right) \cong H_{\mathfrak{m}}^{n-i}(S)$. Since $S$ satisfies the vanishing conditions for maps of Tor, by considering the map $A \rightarrow R \rightarrow S$, we have $\operatorname{Tor}_{i}^{A}\left(E_{A}, R\right) \rightarrow \operatorname{Tor}_{i}^{A}\left(E_{A}, S\right)$ vanishes for every $i \geq 1$. Hence $H_{\mathfrak{m}}^{j}(R) \rightarrow H_{\mathfrak{n}}^{j}(S)$ vanishes for $j<n=\operatorname{dim} R$. The last assertion then follows from Lemma 3.3 .

Remark 3.5. We assume $(S, \mathfrak{n})$ is complete in the proof of Proposition 3.4 because we use Cohen's structure theorem to find $A \rightarrow R$ module-finite with $A$ regular. Hence the conclusion of Proposition 3.4 still holds when we work with rings that are essentially of finite type over a field (we can use Noether normalization instead). 


\section{VAnishing CONDitions FOR MAPS OF TOR AND THE SPLitTing PROPERTy}

The goal of this section is to prove $(1) \Leftrightarrow(3)$ in Theorem 1.2. As a corollary we will see that if $S$ satisfies the vanishing conditions for maps of Tor then $S$ is a splinter. We start with a lemma by restating (4.5) in [HH95]. This was stated only in the complete case in [HH95], however the same argument works for rings essentially of finite type over a field (one needs to replace Cohen's structure theorem by Noether normalization in [HH95]).

Lemma 4.1 ( $c f .(4.5)$ in [HH95]). Let $(S, \mathfrak{n})$ be either complete or essentially of finite type over a field. To show $(S, \mathfrak{n})$ satisfies the vanishing conditions for maps of Tor in a given characteristic, we may assume $A$ is local, $R$ is a domain, $A \rightarrow S$ is surjective and $M$ is finitely generated. Furthermore, it suffices to prove the vanishing of Tor for $i=1$.

Remark 4.2. Suppose $A \rightarrow R \rightarrow S$ satisfies that $A \rightarrow R$ is module-finite and torsion-free, and the composite map $A \rightarrow S$ is surjective. In this situation, if we set $S=A / P=R / \widetilde{P}$, then modulo $\widetilde{P}$, elements of $R$ come from elements of $A$. Thus in this case $R=A+\widetilde{P}$.

Theorem 4.3. Let $(S, \mathfrak{n})$ be either complete or essentially of finite type over a field.

(1) $S$ satisfies the vanishing conditions for maps of Tor.

(2) For every regular local ring $A$ with $S=A / P$, and every module-finite torsion-free extension $A \rightarrow B$ with $Q \in \operatorname{Spec} B$ lying over $P, P \rightarrow Q$ splits as $A$-modules.

(3) For every regular local ring $A$ with $S=A / P$, every module-finite torsion-free extension $A \rightarrow B$ that splits as $A$-modules, and every $Q \in$ Spec $B$ lying over $P$, the map $P \rightarrow Q$ splits as $A$-modules.

Then we have $(2) \Rightarrow(1) \Rightarrow(3)$. In particular, $(1) \Leftrightarrow(2) \Leftrightarrow(3)$ in equal characteristic.

Proof. Let $S=A / P$ with $A$ regular. Let $A \rightarrow B$ be a module-finite torsion-free extension with $Q \in \operatorname{Spec} B$ lying over $P$. We form the $\operatorname{ring} R_{0}=A+Q \subseteq B$. Then $R_{0}$ is also a module-finite torsion-free extension of $A$ and we have $R_{0} / Q=A / P=S$. Now we look at the following commutative diagram:

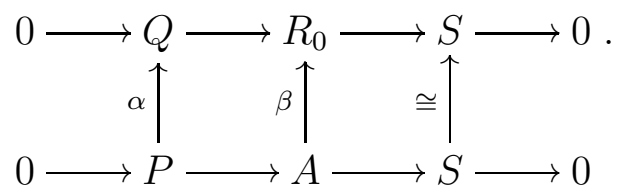

Tensoring the above diagram with an arbitrary $A$-module $M$, we get:

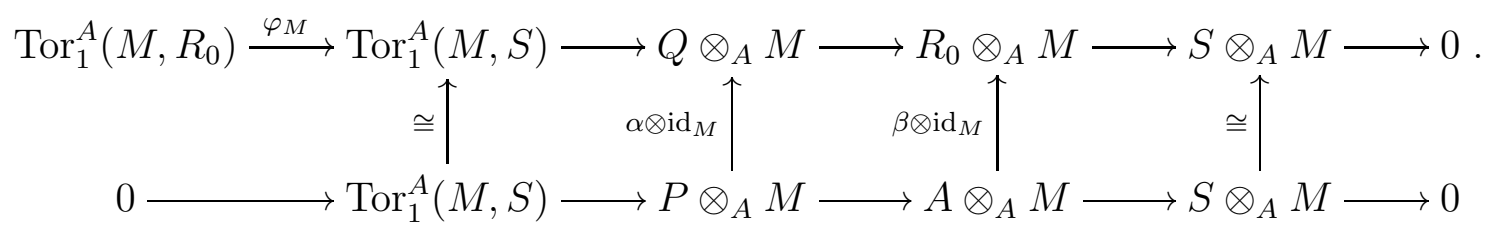

By a diagram chasing, one can see that

$$
\alpha \otimes \mathrm{id}_{M} \text { is injective } \Longleftrightarrow \varphi_{M}=0 \text { and } \beta \otimes \mathrm{id}_{M} \text { is injective }
$$

$(1) \Rightarrow(3)$ : Suppose $A \rightarrow B$ splits (i.e., we are in condition (3)), then $A \rightarrow R_{0}$ also splits, in particular $\beta \otimes \operatorname{id}_{M}$ is injective. Applying the vanishing conditions for maps of Tor to $A \rightarrow R_{0} \rightarrow S$, we know $\varphi_{M}=0$. Now (4.3.1) implies $P \otimes_{A} M \stackrel{\alpha \otimes \mathrm{id}_{M}}{\longrightarrow} Q \otimes_{A} M$ is injective for 
every $M$. But this implies $P \rightarrow Q$ splits by Corollary 5.2 in [HR76] since $Q / P$ is a finitely generated $A$-module.

$(2) \Rightarrow(1)$ : By Lemma 4.1, we may assume $A \rightarrow R \rightarrow S$ satisfies $A \rightarrow S$ is surjective. Now we set $B=R$ and $Q=\operatorname{ker}(R \rightarrow S)$ in the above discussion. By Remark 4.2, we have $Q=\widetilde{P}$ and hence $R=A+\widetilde{P}=A+Q=R_{0}$ in this situation. Now (2) implies $P \rightarrow Q$ splits, in particular $\alpha \otimes \mathrm{id}_{M}$ is injective. Thus (4.3.1) implies

$$
\varphi_{M}: \operatorname{Tor}_{1}^{A}(M, R)=\operatorname{Tor}_{1}^{A}\left(M, R_{0}\right) \rightarrow \operatorname{Tor}_{1}^{A}(M, S)
$$

vanishes for every $M$. This proves $S$ satisfies the vanishing conditions for maps of Tor, since it is enough to check the vanishing of Tor for $i=1$ by Lemma 4.1.

Finally, in equal characteristic, every module-finite extension $A \rightarrow B$ splits when $A$ is regular. So $(2) \Leftrightarrow(3)$ and hence $(1) \Leftrightarrow(2) \Leftrightarrow(3)$.

We next prove a lemma.

Lemma 4.4. Let $A \rightarrow B$ be a module-finite extension. Suppose $Q \in$ Spec $B$ lies over $P \in \operatorname{Spec} A$. If $P \rightarrow Q$ splits as $A$-modules and $\operatorname{depth}_{P} A \geq 2$, then $A \rightarrow B$ splits compatibly with $P \rightarrow Q$, i.e., there exists a splitting $\theta: B \rightarrow A$ such that $\theta(Q)=P$. In particular, $A / P \rightarrow B / Q$ splits as $A$-modules.

Proof. Let $\phi: Q \rightarrow P$ be a splitting. The exact sequences $0 \rightarrow Q \rightarrow B \rightarrow B / Q \rightarrow 0$ and $0 \rightarrow P \rightarrow A$ induce a commutative diagram:

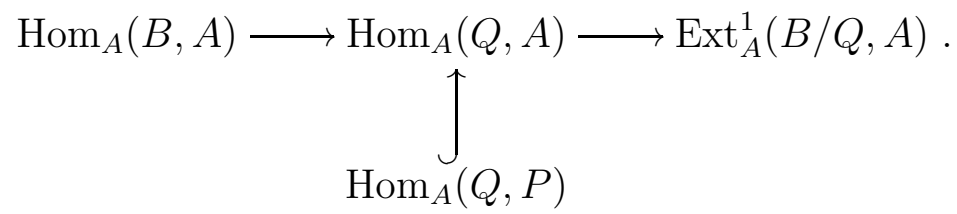

Since $B / Q$ is a finitely generated $A$-module annihilated by $P$ and $\operatorname{depth}_{P} A \geq 2$, we know that $\operatorname{Ext}_{A}^{1}(B / Q, A)=0$ by Proposition 18.4 in Eis95. Hence $\operatorname{Hom}_{A}(B, A)$ maps onto $\operatorname{Hom}_{A}(Q, A)$, in particular it maps onto the image of $\operatorname{Hom}_{A}(Q, P)$. Thus there is a map $\theta$ : $B \rightarrow A$ such that $\left.\theta\right|_{Q}=\phi$. We show that $\theta$ has to be a splitting from $B$ to $A$. Suppose $\theta(1)=a \in A$, for every nonzero element $r \in P$, we have

$$
r a=r \theta(1)=\theta(r)=\phi(r)=r .
$$

So $a=1$ and hence $\theta$ is a splitting from $B$ to $A$ such that $\theta(Q)=P$, i.e., $\theta$ compatibly splits $P \rightarrow Q$. Finally $\bar{\theta}$ gives a splitting from $B / Q \rightarrow A / P$. This finishes the proof.

Corollary 4.5. If $S$ satisfies the vanishing conditions for maps of Tor, then $S$ is a splinter.

Proof. We use a construction similar to the one used in the proof of Lemma 3.1. We write $S=A / P$ such that $A$ is a regular local ring with $\operatorname{depth}_{P} A \geq 2$ (this can be achieved, for example, by adding indeterminants). Let $S \rightarrow T$ be a module-finite domain extension. Let $t_{1}, \ldots, t_{n}$ be a set of generators of $T$ over $S=A / P$. Each $t_{i}$ satisfies a monic polynomial $f_{i}$ over $S$. We lift each $f_{i}$ to $A$ and form the ring $B=\frac{A\left[x_{1}, \ldots, x_{n}\right]}{\left(f_{1}, \ldots, f_{n}\right)}$. We have a natural surjection $B \rightarrow T$ with kernel $Q \in \operatorname{Spec} B$. It is straightforward to check that $Q$ lies over $P$.

Since $B$ is finite free over $A$, we know that $A \rightarrow B$ splits as a map of $A$-modules. Now applying $(1) \Rightarrow(3)$ of Theorem 4.3, $P \rightarrow Q$ is split. Since $\operatorname{depth}_{P} A \geq 2$, by Lemma 4.4, $S=A / P \rightarrow B / Q=T$ splits as a map of $A$-modules (hence also as a map of $S$-modules). As this is true for any module-finite domain extension $T$ of $S, S$ is a splinter. 
Remark 4.6. Applying Corollary 4.5 with $S$ a regular local ring, we see that the vanishing conjecture for maps of Tor implies the direct summand conjecture in all characteristics. Although this is well known, we want to point out that the original proof given in [HH95] and [Ran00] depends on applying the vanishing conjecture to the map $A \rightarrow R \rightarrow S=R / \mathfrak{m}$, i.e., studying the map from a mixed characteristic $\operatorname{ring}(R, \mathfrak{m})$ to its residue field $S=R / \mathfrak{m}$. Such a map, though being very natural, does not preserve the characteristic of the rings! Our above theorem gives a totally different proof, and it shows that the vanishing conjecture for maps of Tor, even if we restrict to $A \rightarrow R \rightarrow S$ all of the same characteristic, still implies the direct summand conjecture.

\section{MAin RESUlts}

In this section we prove our main theorem in equal characteristic. We begin by recalling some facts about dualizing complexes. For an integral scheme $X$, a normalized dualizing complex $\omega_{X}^{\bullet}$ is an object in $D_{\text {Coh }}^{b}(X)$ which has finite injective dimension, such that the canonical map $O_{X} \rightarrow \mathbf{R} \mathscr{H} \mathrm{om}_{X}\left(\omega_{X}^{\bullet}, \omega_{X}^{\bullet}\right)$ is an isomorphism in $D_{\mathrm{Coh}}^{b}(X)$, and the first nonzero cohomology of $\omega_{X}^{\bullet}$ lies in degree $-\operatorname{dim} X$. Note that under this definition, if $\omega_{X}^{\bullet}$ is a normalized dualizing complex, then so is $\omega_{X}^{\bullet} \otimes \mathscr{L}$ for any line bundle $\mathscr{L}$ (in fact this is all the ambiguity for a connected scheme [Har66]).

To clear this ambiguity, notice that all our rings and schemes in this section are essentially of finite type over a field $k$ (or over a fixed scheme Spec $S$ as in Theorem 5.11 and Remark 5.13). Therefore we simply define $\omega_{X}^{\bullet}=\pi^{!} k$ (resp., $\pi^{!} \omega_{S}^{\bullet}$ for some chosen $\omega_{S}^{\bullet}$ ), where $\pi: X \rightarrow$ Spec $k$ (resp., $X \rightarrow \operatorname{Spec} S$ ) is the structural map and $\pi^{!}$is the functor from Grothendieck duality theory [Har66]. By standard duality theory, $\omega_{X}^{\bullet}$ is a normalized dualizing complex discussed in the previous paragraph. Moreover, after this choice, we have

$$
\mathbf{R} \mathscr{H}_{\mathrm{om}_{X}}\left(\mathbf{R} f_{*} O_{Y}, \omega_{X}^{\bullet}\right) \cong \mathbf{R} f_{*} \omega_{Y}^{\bullet}
$$

for any proper and dominant morphism of integral schemes $f: Y \rightarrow X$, where $X, Y$ are both essentially of finite type over a field $k$ or over a fixed scheme Spec $S$. We refer to [Har66] for details on Grothendieck duality theory and to Section 2.3 in [BST15] for a very nice summary.

Next we recall that for an excellent local domain $A$, a complex

$$
F_{\bullet}=0 \rightarrow A^{b_{n}} \stackrel{\alpha_{n}}{\longrightarrow} A^{b_{n-1}} \stackrel{\alpha_{n-1}}{\longrightarrow} \cdots \stackrel{\alpha_{2}}{\longrightarrow} A^{b_{1}} \stackrel{\alpha_{1}}{\longrightarrow} A^{b_{0}} \rightarrow 0
$$

of finitely generated free $A$-modules is said to satisfy the standard conditions for rank and height (resp. rank and depth) if, for every $1 \leq i \leq n$, rank $\alpha_{i}+\operatorname{rank} \alpha_{i+1}=b_{i}$ and the height (resp. the depth) of the ideal $I_{\operatorname{rank} \alpha_{i}}\left(\alpha_{i}\right)$ is at least $i$ where $I_{t}\left(\alpha_{i}\right)$ denotes the ideal generated by the size $t$ minors of a matrix for $\alpha_{i}$ : it is independent of the choice of bases for $F_{i}$ and $F_{i-1}$ (rank $\alpha_{i}$ is the largest integer $r$ such that $I_{r}\left(\alpha_{i}\right) \neq 0$ ). We use the convention that $I_{0}\left(\alpha_{i}\right)=R$ and the unit ideal has height infinity.

Remark 5.1. Let $F_{\bullet}$ be a complex of finite free $A$-modules. It is well known that $F_{\bullet}$ is acyclic (which means $F_{\bullet}$ is exact except possibly in degree 0 ) if and only if $F_{\bullet}$ satisfies the standard conditions on rank and depth. Moreover, in characteristic $p>0, F_{\bullet}$ satisfies the standard conditions for rank and height if and only if $F_{\bullet}$ is phantom acyclic. We refer to [HH93] and Abe94 for details on phantom homology. 
Now we are ready to state and prove our key theorem that implies (and is in fact much stronger than) $(2) \Rightarrow(1)$ of Theorem 1.2 .

Theorem 5.2 (Key Theorem). Let $(A, \mathfrak{m})$ be a local domain that is essentially of finite type over a field and $F_{\bullet}: 0 \rightarrow F_{n} \rightarrow \cdots \rightarrow F_{1} \rightarrow F_{0} \rightarrow 0$ be a complex of finitely generated free A-modules that satisfies the standard conditions on rank and height. Let $X \stackrel{f_{0}}{\rightarrow} Y \stackrel{f}{\rightarrow} \operatorname{Spec} A$ be maps of integral schemes such that $Y \rightarrow \operatorname{Spec} A$ is proper surjective and $X$ is a derived splinter. Then the natural map

$$
h^{-i}\left(F_{\bullet} \otimes \mathbf{R} f_{*} O_{Y}\right) \rightarrow h^{-i}\left(F_{\bullet} \otimes \mathbf{R} f_{*} \mathbf{R} f_{0_{*}} O_{X}\right)
$$

induced by the pull back $f_{0}^{*}$ is the zero map for every $i>0$ (note that $F_{i}$ has cohomological degree $-i$ ).

Proof. As the methods we use in characteristic 0 and $p>0$ are very different, we separate the proof in two parts.

Proof in characteristic 0: We assume $(A, \mathfrak{m})$ is of equal characteristic 0 . Let $p: Z \rightarrow Y$ be a resolution of singularities and let $W=X \times_{Y} Z$. We have the following diagram:

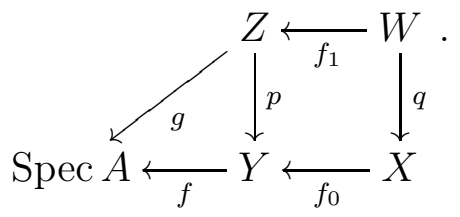

Since $p$ is a resolution of singularities, the map $q$ obtained by base change is proper and surjective. Because $X$ is a derived splinter, the natural map $q^{*}: O_{X} \rightarrow \mathbf{R} q_{*} O_{W}$ has a splitting $\eta$ in the derived category $D(\operatorname{Coh}(X))$, i.e., $\eta \circ q^{*}=$ id. Therefore we have the following commutative diagram in $D(\mathrm{Q} \operatorname{Coh}(\operatorname{Spec} A))$ :

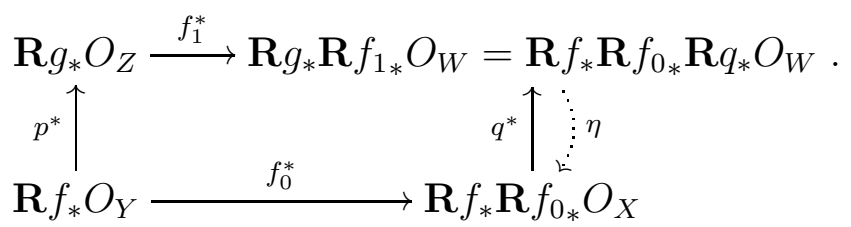

Now we tensor the above diagram with $F_{\bullet}$ in $D(\mathrm{QCoh}(\operatorname{Spec} A))$ and take cohomology in negative degree, we get a commutative diagram (since $F_{\bullet}$ is a complex of free $A$-modules, $\otimes \mathbf{L}$ is the same as $\otimes)$ :

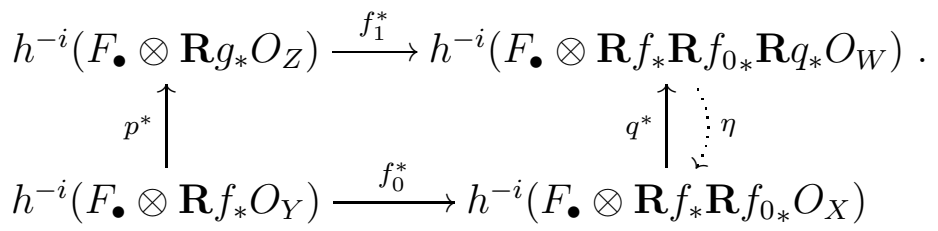

Since $\eta \circ q^{*}=\mathrm{id}$, in order to show $f_{0}^{*}$ induces the zero map, it is enough to show $\eta \circ f_{1}^{*} \circ p^{*}$ induces the zero map by the above commutative diagram. We will show this by proving that $h^{-i}\left(F_{\bullet} \otimes \mathbf{R} g_{*} O_{Z}\right)=0$ for every $i>0$, when $F_{\bullet}$ satisfies the standard conditions for rank and height and $Z \rightarrow \operatorname{Spec} A$ is proper surjective with $Z$ smooth.

We use induction on the dimension of $A$. When $\operatorname{dim} A=0,(A, \mathfrak{m})$ is Artinian and it is easy to see that every complex $F$ • that satisfies the standard conditions on rank and height is split 
exact except at the zeroth spot. Hence the complex $F_{\bullet} \otimes \mathbf{R} g_{*} O_{Z} \cong A^{n} \otimes \mathbf{R} g_{*} O_{Z}=\oplus^{n} \mathbf{R} g_{*} O_{Z}$ has no negative degree part, so $h^{-i}\left(F_{\bullet} \otimes \mathbf{R} g_{*} O_{Z}\right)=0$ for every $i>0$.

Now let $\operatorname{dim} A=d$. We set $G^{\bullet}=F \bullet \otimes \mathbf{R} g_{*} O_{Z}$. Let $\underline{x}=x_{1}, \ldots, x_{d}$ denote a full system of parameters of $A$ and let $C^{\bullet}(\underline{x}, A)$ be the Čech complex associated to $\underline{x}$. We also let

$$
\widetilde{G}^{\bullet}=G^{\bullet} \otimes^{\mathbf{L}} C^{\bullet}(\underline{x}, A)=G^{\bullet} \otimes C^{\bullet}(\underline{x}, A) .
$$

We compute $h^{-i}\left(\widetilde{G}^{\bullet}\right)$ using spectral sequences of the double complex:

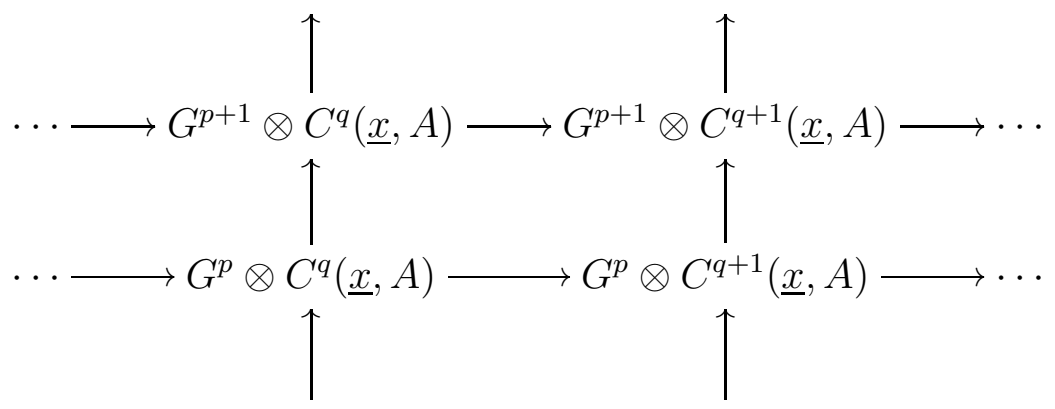

Each $C^{q}(\underline{x}, A)$ is a direct sum of localizations of $A$, in particular it is flat over $A$. So when we take the cohomology of the columns, we get

$$
E_{1}^{p q}=h^{p}\left(G^{\bullet}\right) \otimes C^{q}(\underline{x}, A) .
$$

Note that, when $q=0$, this is just $h^{p}\left(G^{\bullet}\right)$ while when $q>0$, this is a direct sum of proper localizations of $h^{p}\left(G^{\bullet}\right)$. However, when $p<0, h^{p}\left(G^{\bullet}\right)=h^{p}\left(F_{\bullet} \otimes \mathbf{R} g_{*} O_{Z}\right)$ is supported only at the maximal ideal $\mathfrak{m}$ by the induction hypothesis. Because if it is supported at another prime ideal, say $P$, then $h^{p}\left(\left(F_{\bullet}\right)_{P} \otimes \mathbf{R} g_{*} O_{Z \times_{\text {Spec } R} \operatorname{Spec} R_{P}}\right) \neq 0$. But $\left(F_{\bullet}\right)_{P}$ satisfies the standard conditions for rank and height as a free complex over $R_{P}$ (the ranks of all the $F_{i}$ are preserved and the height of an ideal does not decrease when we localize) and $Z \times_{\text {Spec } R}$ Spec $R_{P}$ is smooth, we thus got a contradiction since $\operatorname{dim} R_{P}<d$. Hence we know that $E_{1}^{p q}=0$ when $p<0$ and $q>0$. In sum, the $E_{1}$-stage of the spectral sequence looks like:

$$
\begin{gathered}
E_{1}^{00}=h^{0}\left(G^{\bullet} \longrightarrow E_{1}^{01} \longrightarrow E_{1}^{02} \longrightarrow E_{1}^{-1,1}=0 \longrightarrow E_{1}^{-1,2}=0 \longrightarrow \cdots \longrightarrow E_{1}^{0 d}\right. \\
E_{1}^{-1,0}=h^{-1}\left(G^{\bullet}\right) \longrightarrow 0 \\
E_{1}^{-2,0}=h^{-2}\left(G^{\bullet} \longrightarrow E_{1}^{-2,1}=0 \longrightarrow E_{2}^{-2,2}=0 \longrightarrow \cdots \longrightarrow E_{1}^{-2, d}=0\right.
\end{gathered}
$$

From this we know that

$$
h^{-i}\left(\widetilde{G}^{\bullet}\right)=\underset{12}{E_{1}^{-i, 0}}=h^{-i}\left(G^{\bullet}\right)
$$


for every $i>0$. This is because $E_{1}^{-i, 0}=h^{-i}\left(G^{\bullet}\right)$ is the only nontrivial term that contributes to $h^{-i}\left(\widetilde{G}^{\bullet}\right)$ when $i>0$, and all the further differentials at this spot:

$$
E_{r}^{-i+r-1,-r} \rightarrow E_{r}^{-i, 0} \rightarrow E_{r}^{-i-r+1, r}
$$

vanish since $E_{r}^{-i+r-1,-r}=E_{r}^{-i-r+1, r}=0$ when $i>0$ and $r \geq 1$.

Rewriting (5.2.1), we have:

$$
h^{-i}\left(F_{\bullet} \otimes \mathbf{R} g_{*} O_{Z}\right)=h^{-i}\left(F_{\bullet} \otimes \mathbf{R} g_{*} O_{Z} \otimes C^{\bullet}(\underline{x}, A)\right) .
$$

Since we have functorial isomorphism $\mathbf{R} \Gamma_{\mathfrak{m}}(-) \stackrel{\cong}{\rightarrow} C^{\bullet}(\underline{x}, A) \otimes-$ in $D(\mathrm{QCoh}(\operatorname{Spec} A))$ by Proposition 3.1.2 in [Lip02]. The above yields:

$$
h^{-i}\left(F_{\bullet} \otimes \mathbf{R} g_{*} O_{Z}\right) \cong h^{-i}\left(F_{\bullet} \otimes \mathbf{R} \Gamma_{\mathfrak{m}} \mathbf{R} g_{*} O_{Z}\right) .
$$

By local duality, we have that

$$
h^{j}\left(\mathbf{R} \Gamma_{\mathfrak{m}} \mathbf{R} g_{*} O_{Z}\right)=h^{-j}\left(\mathbf{R} \operatorname{Hom}\left(\mathbf{R} g_{*} O_{Z}, \omega_{A}^{\bullet}\right)\right)^{\vee}=h^{-j}\left(\mathbf{R} g_{*} \omega_{Z}^{\bullet}\right)^{\vee}
$$

where $\omega_{A}^{\bullet}, \omega_{Z}^{\bullet}$ are the normalized dualizing complexes of $\operatorname{Spec} A$ and $Z$. Since $Z$ is smooth, $\omega_{Z}^{\bullet} \cong \omega_{Z}[n]$ where $n=\operatorname{dim} Z$. Hence $h^{-j}\left(\mathbf{R} g_{*} \omega_{Z}^{\bullet}\right)=h^{n-j}\left(\mathbf{R} g_{*} \omega_{Z}\right)=0$ when $n-j>n-d$ (equivalently, $j<d$ ) by Theorem 2.1 in [Kol86] 5 Now from (5.2.4), we know that

$$
h^{j}\left(\mathbf{R} \Gamma_{\mathfrak{m}} \mathbf{R} g_{*} O_{Z}\right)=0, \forall j<d .
$$

On the other hand, we know that $F$. satisfies the standard conditions for rank and height. This implies $I_{\operatorname{rank} \alpha_{n}}\left(\alpha_{n}\right)$ must be the unit ideal when $n>d$ because there are no proper ideals in $R$ with height strictly bigger than the dimension of $R$. From this it follows that $F$. is split exact at cohomological degree $-n$ when $n>d$ by Lemma 1 in [BE73]. Therefore, in $D(\operatorname{Coh}(\operatorname{Spec} A))$ or $D(\mathrm{QCoh}(\operatorname{Spec} A)), F_{\bullet}$ is quasi-isomorphic to a free complex

$$
H_{\bullet}: 0 \rightarrow H_{k} \rightarrow H_{k-1} \rightarrow \cdots \rightarrow H_{1} \rightarrow H_{0} \rightarrow 0
$$

with $k \leq d$ and $H_{j}$ has cohomological degree $-j$. Now from (5.2.5), it is straightforward to check that

$$
h^{-i}\left(F_{\bullet} \otimes \mathbf{R} \Gamma_{\mathfrak{m}} \mathbf{R} g_{*} O_{Z}\right)=h^{-i}\left(H_{\bullet} \otimes \mathbf{R} \Gamma_{\mathfrak{m}} \mathbf{R} g_{*} O_{Z}\right)=0
$$

for every $i>0$. Hence by (5.2.3) we know that $h^{-i}\left(F_{\bullet} \otimes \mathbf{R} g_{*} O_{Z}\right)=0$ for every $i>0$. This finishes our proof in equal characteristic 0.

Proof in characteristic $p>0$ : Now we assume $(A, \mathfrak{m})$ is of equal characteristic $p>0$. By Theorem 1.5 in [Bha12], there exists a finite surjective morphism $\pi: Z \rightarrow Y$ such that the pullback $\pi_{\geq 1}^{*}: \tau_{\geq 1} \mathbf{R} f_{*} O_{Y} \rightarrow \tau_{\geq 1} \mathbf{R} f_{*} \pi_{*} O_{Z}$ is the zero map. From this it follows (see the proof of Theorem 1.4 in [Bha12] or Lemma 5.1 in [BST15]) that the natural map $\mathbf{R} f_{*} O_{Y} \rightarrow \mathbf{R} f_{*} \pi_{*} O_{Z}$ factors through

$$
\mathbf{R} f_{*} O_{Y} \stackrel{\theta}{\rightarrow}(f \circ \pi)_{*} O_{Z} \stackrel{\iota}{\rightarrow} \mathbf{R} f_{*} \pi_{*} O_{Z}
$$

${ }^{5}$ In [Kol86], the main theorem requires that the schemes are projective, but this is not essential. One can refer to Corollary 6.11 in EV92. 
Let $g=f \circ \pi$. We know that $g_{*} O_{Z}$ is a module-finite extension of $A$, as $Z \rightarrow \operatorname{Spec} A$ is proper. Let $W=Z \times_{Y} X$. We have the following commutative diagram:

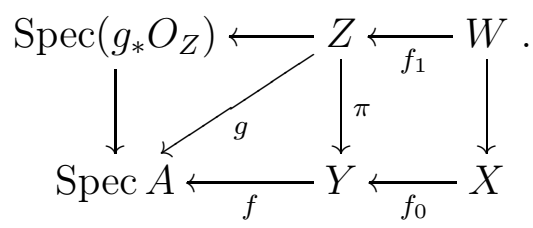

This together with (5.2.6) tell us that there is a commutative diagram in $D(\mathrm{QCoh}(\operatorname{Spec} A))$ :

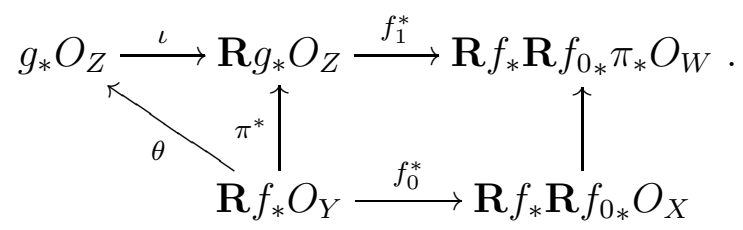

Now we pick an arbitrary element $x \in h^{-i}\left(F_{\bullet} \otimes \mathbf{R} f_{*} O_{Y}\right)$ for an arbitrary $i>0$. We want to show that $x$ maps to 0 in $h^{-i}\left(F_{\bullet} \otimes \mathbf{R} f_{*} \mathbf{R} f_{0_{*}} O_{X}\right)$. To prove this, we first look at the image of $x$ under the map

$$
h^{-i}\left(F_{\bullet} \otimes \mathbf{R} f_{*} O_{Y}\right) \stackrel{\theta}{\rightarrow} h^{-i}\left(F_{\bullet} \otimes g_{*} O_{Z}\right) .
$$

Let $y=\theta(x)$. Since we are in equal characteristic $p>0$ and $A \rightarrow g_{*} O_{Z}$ is a modulefinite extension, $A^{+}=\left(g_{*} O_{Z}\right)^{+}$is a balanced big Cohen-Macaulay algebra over $A$ [HH92].

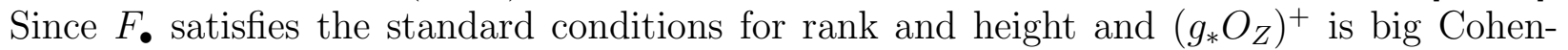
Macaulay, it follows from the generalized Buchsbaum-Eisenbud criterion (see Theorem 1.2.3 in [Abe94]) that $h^{-i}\left(F_{\bullet} \otimes\left(g_{*} O_{Z}\right)^{+}\right)=0$ for $i>0$. In particular, we know that there exists a module-finite extension $B$ of $g_{*} O_{Z}$ such that the image of $y$ in $h^{-i}\left(F_{\bullet} \otimes B\right)$ is 0 . Let $W^{\prime}=W \times_{\operatorname{Spec}\left(g_{*} O_{Z}\right)} \operatorname{Spec} B$. We know $\pi^{\prime}: W^{\prime} \rightarrow W \rightarrow X$ is a finite surjective map of schemes. Since $X$ is a derived splinter, in particular a splinter, we know that $O_{X} \rightarrow \pi_{*}^{\prime} O_{W^{\prime}}$ has a splitting $\eta$. In sum, after tensoring (5.2.7) with $F_{\bullet}$ in $D(\mathrm{Q} \operatorname{Coh}(\operatorname{Spec} A))$ and taking cohomology, we get a commutative diagram:

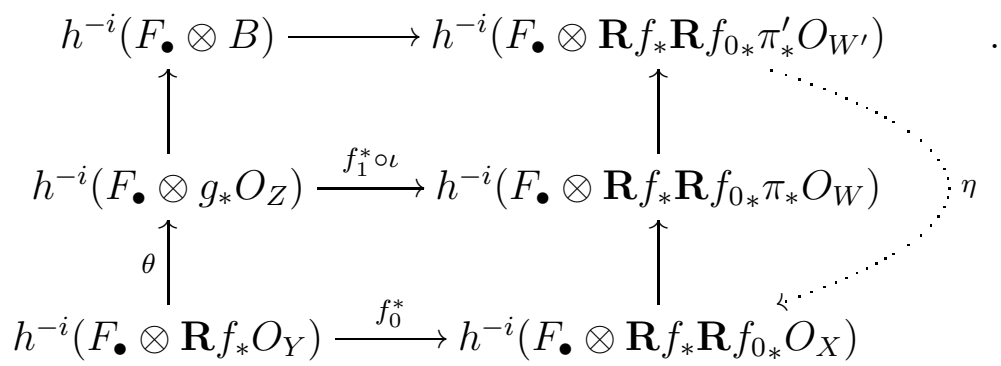

From this diagram, it is easy to see that the image of $x \in h^{-i}\left(F_{\bullet} \otimes \mathbf{R} f_{*} O_{Y}\right)$ maps to 0 under $f_{0}^{*}$, because by our construction, the image of $x$ in $h^{-i}\left(F_{\bullet} \otimes B\right)$ is 0 . Since our choice of $x$ and $i>0$ are arbitrary, this proves that the map $f_{0}^{*}: h^{-i}\left(F_{\bullet} \otimes \mathbf{R} f_{*} O_{Y}\right) \rightarrow h^{-i}\left(F_{\bullet} \otimes \mathbf{R} f_{*} \mathbf{R} f_{0_{*}} O_{X}\right)$ is the zero map for every $i>0$. This finishes our proof in equal characteristic $p>0$.

Remark 5.3. Note that in the above proof, in equal characteristic $p>0$, we only need to assume $X$ is a splinter. But splinters and derived splinters are the same in characteristic $p>0$ [Bha12. In fact, in the course of our proof we use Theorem 1.5 of [Bha12, which 
is a key ingredient in proving splinters and derived splinters are equivalent in characteristic $p>0$. We refer to Bha12 for details.

In the case of maps between rings instead of schemes, our Key Theorem 5.2 specializes to the following corollary:

Corollary 5.4. Let $(A, \mathfrak{m})$ be a local domain that is essentially of finite type over a field and let $M$ be a finitely generated $A$-module of finite projective dimension. Let $A \rightarrow R \rightarrow S$ be ring homomorphisms such that $A \rightarrow R$ is a module-finite domain extension and $S$ is a derived splinter. Then the natural map:

$$
\operatorname{Tor}_{i}^{A}(M, R) \rightarrow \operatorname{Tor}_{i}^{A}(M, S)
$$

is the zero map for every $i>0$.

Proof. Since $M$ has a finite projective dimension, it has a finite free resolution $F_{\bullet}$. As $F_{\bullet}$ is acyclic, it satisfies the standard conditions for rank and depth and hence also the standard conditions for rank and height. Applying Theorem 5.2 to $F_{\bullet}, Y=\operatorname{Spec} R, X=\operatorname{Spec} S$ and noticing that there are no higher direct images because all the maps are affine, we find that $h^{-i}\left(F_{\bullet} \otimes R\right) \rightarrow h^{-i}\left(F_{\bullet} \otimes S\right)$ vanishes for every $i>0$. $\operatorname{But}^{\operatorname{Tor}_{i}^{A}}(M, R)=h^{-i}\left(F_{\bullet} \otimes R\right)$ and $\operatorname{Tor}_{i}^{A}(M, S)=h^{-i}\left(F_{\bullet} \otimes S\right)$, so the conclusion follows.

Now we state and prove our main theorem.

Theorem 5.5. Let $S$ be a local domain that is essentially of finite type over a field. The following are equivalent:

(1) S satisfies the vanishing conditions for maps of Tor.

(2) $S$ is a derived splinter.

(3) For every regular local ring $A$ with $S=A / P$ and every module-finite torsion-free extension $A \rightarrow B$ with $Q \in \operatorname{Spec} B$ lying over $P, P \rightarrow Q$ splits as $A$-modules.

Proof. We already know $(1) \Leftrightarrow(3)$ by Theorem 4.3, Moreover, recall that derived splinters are the same as rational singularities (equivalently, pseudo-rational singularities) in characteristic 0 , and are equivalent to splinters in characteristic $p>0$. Hence $(1) \Rightarrow(2)$ follows from Remark 3.5 and Corollary 4.5 in characteristic 0 and characteristic $p>0$ respectively. Finally we prove $(2) \Rightarrow(1)$. Suppose we have $A \rightarrow R \rightarrow S$ with $A$ regular and $R$ module-finite and torsion-free over $A$. To check $\operatorname{Tor}_{i}^{A}(M, R) \rightarrow \operatorname{Tor}_{i}^{A}(M, S)$ vanishes, we can assume $A$ is local, $R$ is a domain and $M$ is a finitely generated $A$-module by Lemma 4.1. Since $A$ is regular, $M$ has finite projective dimension. Hence the vanishing of $\operatorname{Tor}_{i}^{A}(M, R) \rightarrow \operatorname{Tor}_{i}^{A}(M, S)$ follows immediately from Corollary 5.4.

Remark 5.6. (1) We point out that in Theorem 5.5, (2) $\Rightarrow(1)$ in characteristic $p>0$ also follows directly from the fact that $R^{+}$is a balanced big Cohen-Macaulay algebra: one can use the same argument as in Theorem 4.1 in [HH95] and simply notice that the map $S \rightarrow S^{+}$is always pure when $S$ is a splinter in characteristic $p>0$.

(2) However, our method in characteristic 0 is of great interest: it gives the first proof of Theorem 1.1 (even in the regular case) in characteristic 0 without using reduction to characteristic $p>0$. In fact, our result in characteristic 0 doesn't even seem to follow from reduction to characteristic $p>0$. It is well known from [Smi97] and Har98 that a local ring essentially of finite type over a field of characteristic 0 has 
rational singularities if and only if its mod $p$ reductions, for all sufficiently large $p$, are $F$-rational. But $F$-rationality is known to be weaker than being a derived splinter in characteristic $p>0$ and hence $F$-rational rings do not satisfy the vanishing conditions for maps of Tor in general by Theorem 5.5.

(3) Moreover, equal characteristic regular local rings satisfying the vanishing conditions for maps of Tor is a very special case of our Key Theorem 5.2, the case that $A$ is

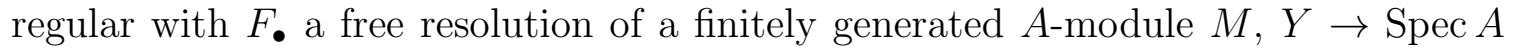
is finite surjective and $X$ is regular affine. So our Theorem 5.2 greatly extends Hochster-Huneke's Theorem 1.1, and actually it also generalizes the main theorems of [HH93].

Remark 5.7. We point out that Boutot's theorem that direct summands of rational singularities are rational singularities [Bou87] follows from our vanishing conditions for maps of Tor applied to $M=E_{A}$, the injective hull of $A$. Let $(R, \mathfrak{m}) \rightarrow(S, \mathfrak{n})$ be a split map of local rings essentially of finite type over a field of characteristic 0 and let $S$ have rational singularities. For every surjection $\left(B, \mathfrak{m}_{1}\right) \rightarrow(R, \mathfrak{m})$ with $B$ equidimensional, we can find $\left(A, \mathfrak{m}_{0}\right) \rightarrow$ $\left(B, \mathfrak{m}_{1}\right)$ module-finite with $\left(A, \mathfrak{m}_{0}\right)$ regular by Noether normalization. Now we consider the map $A \rightarrow B \rightarrow R \rightarrow S$. Since $S$ has rational singularities, it satisfies the vanishing conditions for maps of Tor by Theorem 5.5. Hence $\operatorname{Tor}_{i}^{A}\left(E_{A}, B\right) \rightarrow \operatorname{Tor}_{i}^{A}\left(E_{A}, R\right) \rightarrow \operatorname{Tor}_{i}^{A}\left(E_{A}, S\right)$ vanishes for $i \geq 1$. This implies $\operatorname{Tor}_{i}^{A}\left(E_{A}, B\right) \rightarrow \operatorname{Tor}_{i}^{A}\left(E_{A}, R\right)$ vanishes for $i \geq 1$ because $R \rightarrow S$ splits. Hence $R$ satisfies the vanishing conditions for maps of local cohomology (recall that $\left.\operatorname{Tor}_{i}^{A}\left(E_{A}, B\right)=H_{\mathfrak{m}}^{d-i}(B)\right)$. Therefore by Lemma 3.3, $R$ has rational singularities.

As a consequence of Theorem [5.5, we obtain a new characterization of rational singularities:

Corollary 5.8. Let $(S, \mathfrak{n})$ be a local domain essentially of finite type over a field of characteristic 0 . Then $S$ has rational singularities if and only if for every regular local ring $A$ with $S=A / P$, every module-finite torsion-free extension $A \rightarrow T$, and every $Q \in$ Spec $T$ lying over $P$, the map $P \rightarrow Q$ splits as a map of $A$-modules.

Proof. This follows immediately from $(2) \Leftrightarrow(3)$ in Theorem 5.5, and the result that derived splinters are exactly rational singularities in equal characteristic 0 .

We next want to prove a theorem that characterizes the vanishing conditions for maps of local cohomology in equal characteristic. We first prove a lemma that is of independent interest. Recall that in characteristic $p>0,0_{H_{\mathfrak{n}}^{d}(S)}^{*}$ (the tight closure of 0 ) is the largest proper submodule of $H_{\mathfrak{n}}^{d}(S)$ that is stable under the natural Frobenius action [Smi97.

Lemma 5.9. Let $(S, \mathfrak{n})$ be a local domain of equal characteristic $p>0$. Then we have:

$$
\sum_{R} \operatorname{im}\left(H_{\mathfrak{m}}^{d}(R) \rightarrow H_{\mathfrak{n}}^{d}(S)\right)=0_{H_{\mathfrak{n}}^{d}(S)}^{*}
$$

where the sum is taken over all $(R, \mathfrak{m}) \rightarrow(S, \mathfrak{n})$ such that $\operatorname{dim} R / P>\operatorname{dim} S=d$ for every minimal prime $P$ of $R$.

Proof. Take a surjection $(R, \mathfrak{m}) \rightarrow(S, \mathfrak{n})$, we first prove that the image of $H_{\mathfrak{m}}^{d}(R) \rightarrow H_{\mathfrak{n}}^{d}(S)$ is contained in $0_{H_{\mathfrak{n}}^{d}(S)}^{*}$. Since $\operatorname{dim} R / P>d$ for every minimal prime $P$ of $R, R \rightarrow S$ obviously factors through $R \rightarrow R^{\prime} \rightarrow S$ for some domain $R^{\prime}$ with $\operatorname{dim} R^{\prime}=d+1$. Hence the image of $H_{\mathfrak{m}}^{d}(R) \rightarrow H_{\mathfrak{n}}^{d}(S)$ is contained in the image of $H_{\mathfrak{m}}^{d}\left(R^{\prime}\right) \rightarrow H_{\mathfrak{n}}^{d}(S)$, which is clearly a submodule 
of $H_{\mathfrak{n}}^{d}(S)$ stable under the Frobenius action. Thus it is contained in $0_{H_{\mathfrak{n}}^{d}(S)}^{*}$ as long as it is not equal to $H_{\mathfrak{n}}^{d}(S)$. Therefore, it suffices to show that $H_{\mathfrak{m}}^{d}\left(R^{\prime}\right) \rightarrow H_{\mathfrak{n}}^{d}(S)$ is not surjective. Write $S=R^{\prime} / Q$ for some height one prime ideal $Q$ of $R^{\prime}$, we have the long exact sequence of local cohomology:

$$
\cdots \rightarrow H_{\mathfrak{m}}^{d}\left(R^{\prime}\right) \rightarrow H_{\mathfrak{n}}^{d}(S) \rightarrow H_{\mathfrak{m}}^{d+1}(Q) \rightarrow H_{\mathfrak{m}}^{d+1}\left(R^{\prime}\right) .
$$

Since $R^{\prime}$ has dimension $d+1$ and $Q$ is a height one prime, $H_{\mathfrak{m}}^{d+1}(Q) \rightarrow H_{\mathfrak{m}}^{d+1}\left(R^{\prime}\right)$ is not injective by Lemma 3.3 in [Ma14]. Therefore $H_{\mathfrak{m}}^{d}\left(R^{\prime}\right) \rightarrow H_{\mathfrak{n}}^{d}(S)$ is not surjective by (5.9.2). Hence we have proved

$$
\sum_{R} \operatorname{im}\left(H_{\mathfrak{m}}^{d}(R) \rightarrow H_{\mathfrak{n}}^{d}(S)\right) \subseteq 0_{H_{\mathfrak{n}}^{d}(S)}^{*}
$$

On the other hand, Lemma 3.1 shows that that

$$
\sum_{R} \operatorname{im}\left(H_{\mathfrak{m}}^{d}(R) \rightarrow H_{\mathfrak{n}}^{d}(S)\right) \supseteq 0_{H_{\mathfrak{n}}^{d}(S)}^{+}=0_{H_{\mathfrak{n}}^{d}(S)}^{*}
$$

where the last equality follows from the main theorem of [Smi94]. This finishes the proof.

Theorem 5.10. Let $(S, \mathfrak{n})$ be a local domain that is essentially of finite type over a field. In characteristic $0, S$ satisfies the vanishing conditions for maps of local cohomology if and only if $S$ has rational singularities, while in characteristic $p>0, S$ satisfies the vanishing conditions for maps of local cohomology if and only if $S$ is F-rational.

Proof. The characteristic 0 assertion follows (implicitly) from the proof of Theorem 5.5, as $S$ satisfies the vanishing conditions for maps of local cohomology implies $S$ has rational singularities by Lemma 3.3. It remains to prove the characteristic $p>0$ statement. But this follows immediately from Lemma 5.9 and Definition 2.2, since when $S$ is Cohen-Macaulay, $S$ is $F$-rational if and only if $0_{H_{\mathfrak{n}}^{d}(S)}^{*}=0$ [HH94], [Smi97].

Finally, it is quite natural to ask whether our main theorem, Theorem 5.5, holds in mixed characteristic. By Theorem 4.3, (3) $\Rightarrow(1)$ always holds and the main obstruction of $(1) \Rightarrow$ (3) is the direct summand conjecture in mixed characteristic. Below we give a partial answer of $(1) \Rightarrow(2)$. We believe this result and its proof is of independent interest (for example, see Remark 5.12 and 5.13).

Theorem 5.11. If $(S, \mathfrak{n})$ is a quasi-Gorenstein complete local domain (of mixed characteristic) that satisfies the vanishing condition for maps of Tor, then $S$ is a derived splinter.

Proof. We first note that the conditions imply $S$ is Cohen-Macaulay (and thus Gorenstein) by Proposition 3.4 because $S$ is complete and satisfies the vanishing conditions for maps of Tor.

Let $\pi: X \rightarrow$ Spec $S$ be a proper surjective map, we want to show $S \rightarrow \mathbf{R} \pi_{*} O_{X}$ splits in the derived category of $S$-modules. By Chow's Lemma we may assume that $X$ is projective. I claim we may reduce to the case that $X \rightarrow \operatorname{Spec} S$ is a projective and generically finite map between integral schemes 6 We first find an irreducible component $W$ of $X$ and an affine open $U=\operatorname{Spec} B$ of $W$ that dominates $\operatorname{Spec} S$. It follows that $B$ is a domain containing $S$, finitely generated as an $S$-algebra. Let $L$ be the fraction field of $S$, we have $\operatorname{dim}(L \otimes B)=$

${ }^{6}$ This should be well known. We provide the argument because we cannot find a good reference for this in mixed characteristic. 
$\operatorname{dim} B-\operatorname{dim} S$ by Theorem 13.8 in [Eis95]. Hence if $\operatorname{dim} B-\operatorname{dim} S \geq 1$, then $\operatorname{dim}(L \otimes B) \geq 1$. This means there exist nonzero primes in $B$ that contract to 0 in $S$. Pick such a prime $Q$, we have $S \hookrightarrow B / Q$ is injective. Thus $V=\operatorname{Spec} B / Q$ still dominates Spec $S$. Taking the closure of $V$ in $W$, call it $X^{\prime}$, we have $X^{\prime} \rightarrow \operatorname{Spec} S$ is projective and surjective with $\operatorname{dim} X^{\prime}<\operatorname{dim} X$. We could repeat this process until we get $\operatorname{dim} X=\operatorname{dim} S$, i.e., $X \rightarrow \operatorname{Spec} S$ is projective and generically finite. Next we consider the Stein factorization:

$$
X \rightarrow \operatorname{Spec}\left(\pi_{*} O_{X}\right) \rightarrow \operatorname{Spec} S .
$$

Let $T=\pi_{*} O_{X}$. We know that $T$ is a module-finite domain extension of $S$. In particular, since $S$ is complete, this implies $T$ is a local ring and $\mathfrak{n} T$ is primary to the maximal ideal of $T$. The map $X \rightarrow \operatorname{Spec} T$ is projective and birational, thus it is just the blow-up of some ideal $J \subseteq T$. Let $R=T[J t]=T \oplus J t \oplus J^{2} t^{2} \oplus \cdots$ and we have $X=\operatorname{Proj} R$.

Pick $f_{1}, \ldots, f_{n} \in J t=[R]_{1}$ such that $U=\left\{U_{i}=\operatorname{Spec}\left[R_{f_{i}}\right]_{0}\right\}$ is an affine open cover of $X$. We have an exact sequence of chain complexes (see page 150 of [Lip94]):

$$
0 \rightarrow \check{C}^{\bullet}\left(U, O_{X}\right)[-1] \rightarrow\left[C^{\bullet}\left(f_{1}, \ldots, f_{n}, R\right)\right]_{0} \rightarrow T \rightarrow 0 .
$$

Since $\check{C} \bullet\left(U, O_{X}\right) \cong \mathbf{R} \pi_{*} O_{X}$, the above sequence gives us (after rotating) an exact triangle:

$$
\left[\mathbf{R} \Gamma_{R_{>0}} R\right]_{0}=\left[C^{\bullet}\left(f_{1}, \ldots, f_{n}, R\right)\right]_{0} \rightarrow T \rightarrow \mathbf{R} \pi_{*} O_{X} \stackrel{+1}{\longrightarrow}
$$

Applying $\mathbf{R} \Gamma_{\mathfrak{n}}$, we get:

$$
\left[\mathbf{R} \Gamma_{\mathfrak{n}+R>0} R\right]_{0} \rightarrow \mathbf{R} \Gamma_{\mathfrak{n}} T \rightarrow \mathbf{R} \Gamma_{\mathfrak{n}} \mathbf{R} \pi_{*} O_{X} \stackrel{+1}{\rightarrow} .
$$

Let $d=\operatorname{dim} S=\operatorname{dim} T$ and $d+1=\operatorname{dim} R$. Next I prove two claims:

Claim 5.11.2. $\left[H_{\mathfrak{n}+R_{>0}}^{d+1}(R)\right]_{0}=0$, thus $\left[\mathbf{R} \Gamma_{\mathfrak{n}+R_{>0}} R\right]_{0}$ lives in degree $[0,1, \ldots, d]$.

Proof. This is well known, because the $a$-invariant of the Rees ring is always -1 (for example, see 2.4.2 and 2.5.2 of [HS03]). For the sake of completeness we point out that this also follows from (5.11.1). By local duality, the dual of $h^{d}\left(\mathbf{R} \Gamma_{\mathfrak{n}} T\right) \rightarrow h^{d}\left(\mathbf{R} \Gamma_{\mathfrak{n}} \mathbf{R} \pi_{*} O_{X}\right)$ is the natural inclusion $\pi_{*} \omega_{X} \hookrightarrow \omega_{T}$ (since $X \rightarrow \operatorname{Spec} T$ is birational). Hence

$$
\left[H_{\mathfrak{n}+R_{>0}}^{d+1}(R)\right]_{0}=h^{d+1}\left(\left[\mathbf{R} \Gamma_{\mathfrak{n}+R_{>0}} R\right]_{0}\right)=0 .
$$

Claim 5.11.3. There exists an $S$-linear surjection $\phi: T \rightarrow S$ such that the composite:

$$
\left[H_{\mathfrak{n}+R_{>0}}^{d}(R)\right]_{0} \rightarrow H_{\mathfrak{n}}^{d}(T) \stackrel{\phi}{\rightarrow} H_{\mathfrak{n}}^{d}(S)
$$

is the zero map (the first map is induced by the natural surjection $R \rightarrow T$ ).

Proof. Let $R^{\prime}=S \oplus J t \oplus J^{2} t^{2} \oplus \cdots$ be the subring of $R$ (they only differ at the degree 0 spot). We note that since $J$ is a finitely generated $S$-module, $R^{\prime}$ is a Noetherian graded domain over $S$. We have the following commutative diagram:

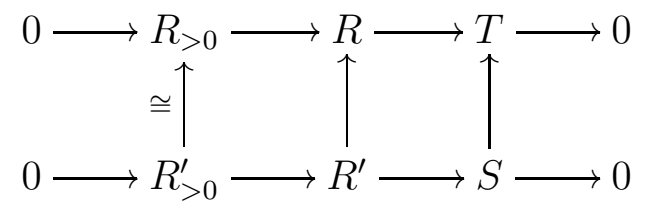


Viewing everything as modules or algebras over $R^{\prime}$, the above diagram induces a commutative diagram of local cohomology:

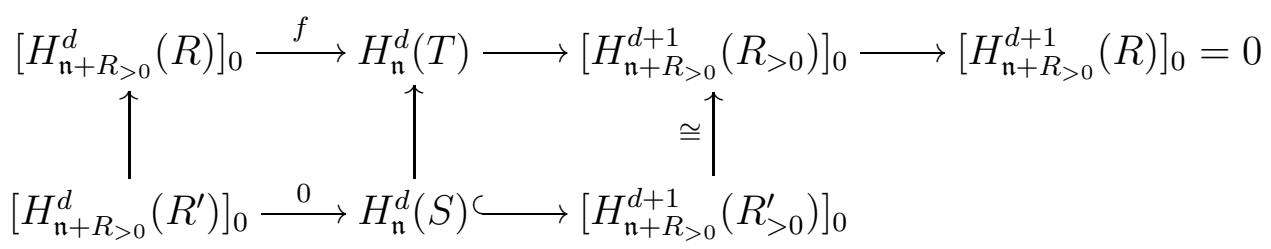

The rightmost 0 on the first line comes from Claim 5.11 .2 and the first map on the second line is 0 because $S$ is complete and satisfies the vanishing conditions for maps of Tor, hence in particular it satisfies the vanishing conditions of local cohomology by Proposition 3.4. Chasing the diagram it follows immediately that $H_{\mathfrak{n}}^{d}(S) \hookrightarrow H_{\mathfrak{n}}^{d}(T) / \operatorname{im}(f)$. Since $S$ is quasiGorenstein, $H_{\mathfrak{n}}^{d}(S) \cong E_{S}$ is an injective $S$-module. So there is a map $g: H_{\mathfrak{n}}^{d}(T) / \operatorname{im}(f) \rightarrow$ $H_{\mathfrak{n}}^{d}(S)$ such that the composite:

$$
H_{\mathfrak{n}}^{d}(S) \rightarrow H_{\mathfrak{n}}^{d}(T) \rightarrow H_{\mathfrak{n}}^{d}(T) / \operatorname{im}(f) \stackrel{g}{\rightarrow} H_{\mathfrak{n}}^{d}(S)
$$

is the identity. In particular, there is an splitting $H_{\mathfrak{n}}^{d}(T) \stackrel{\phi}{\rightarrow} H_{\mathfrak{n}}^{d}(S)$ of $H_{\mathfrak{n}}^{d}(S) \hookrightarrow H_{\mathfrak{n}}^{d}(T)$ such that the composite $\left[H_{\mathfrak{n}+R_{>0}}^{d}(R)\right]_{0} \stackrel{f}{\rightarrow} H_{\mathfrak{n}}^{d}(T) \stackrel{\phi}{\rightarrow} H_{\mathfrak{n}}^{d}(S)$ is the zero map. However, it follows from the following commutative diagram:

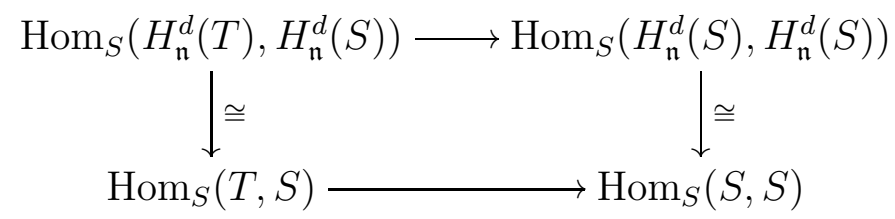

that every splitting $H_{\mathfrak{n}}^{d}(T) \stackrel{\phi}{\rightarrow} H_{\mathfrak{n}}^{d}(S)$ comes from some surjection $T \stackrel{\phi}{\rightarrow} S$.

Now we return to the proof of Theorem 5.11. I claim that the composite map:

$$
\left[\mathbf{R} \Gamma_{\mathfrak{n}+R>0} R\right]_{0} \rightarrow \mathbf{R} \Gamma_{\mathfrak{n}} T \rightarrow H_{\mathfrak{n}}^{d}(T)[-d] \stackrel{\phi}{\rightarrow} H_{\mathfrak{n}}^{d}(S)[-d] \cong \mathbf{R} \Gamma_{\mathfrak{n}} S
$$

is the zero map in the derived category: it induces zero on the $d$-th cohomology by Claim 5.11.3, but by Claim $5.11 .2,\left[\mathbf{R} \Gamma_{\mathfrak{n}+R_{>0}} R\right]_{0}$ lives in degree $[0,1, \ldots, d]$ while $H_{\mathfrak{n}}^{d}(S)[-d]$ lives only in degree $d$, so the map is zero in the derived category. The last isomorphism follows because $S$ is Cohen-Macaulay.

Let $\phi$ be the surjection in Claim 5.11.3. There exits $t \in T$ such that $\phi(t)=1 \in S$, in particular the composite $S \stackrel{\cdot t}{\rightarrow} T \stackrel{\phi}{\rightarrow} S$ is the identity. From the above discussion and (5.11.1), we have a natural diagram in the derived category of $S$-modules:

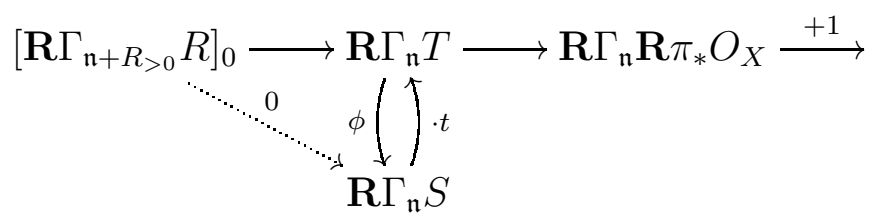


Taking Matlis dual and applying local duality, we get:

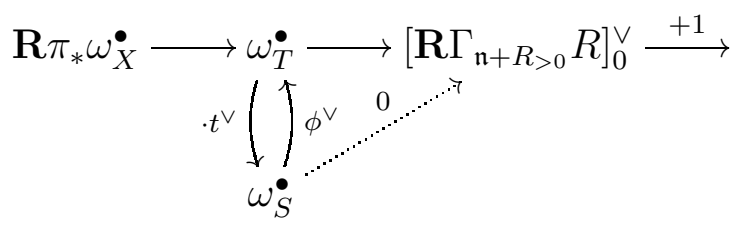

From (5.11.4) it follows that $\phi^{\vee}$ factors through a map $\omega_{S}^{\bullet} \rightarrow \mathbf{R} \pi_{*} \omega_{X}^{\bullet}$ such that the composite:

$$
\omega_{S}^{\bullet} \rightarrow \mathbf{R} \pi_{*} \omega_{X}^{\bullet} \rightarrow \omega_{T}^{\bullet} \stackrel{\cdot t^{\vee}}{\longrightarrow} \omega_{S}^{\bullet}
$$

is the identity. Applying $\mathbf{R} \operatorname{Hom}_{S}\left(-, \omega_{S}^{\bullet}\right)$, we obtain:

$$
S \stackrel{\cdot t}{\rightarrow} T=\pi_{*} O_{X} \rightarrow \mathbf{R} \pi_{*} O_{X} \rightarrow S
$$

such that the composite is the identity. But this implies $S \rightarrow \mathbf{R} \pi_{*} O_{X} \stackrel{\cdot t}{\rightarrow} \mathbf{R} \pi_{*} O_{X} \rightarrow S$ is the identity (the second map is induced by $O_{X} \stackrel{t}{\rightarrow} O_{X}$ viewing $t$ as a section of $X \rightarrow \operatorname{Spec} S$ ). Hence $S \rightarrow \mathbf{R} \pi_{*} O_{X}$ splits in the derived category of $S$-modules. Therefore $S$ is a derived splinter, as desired.

At the moment we don't see how to drop the quasi-Gorenstein hypothesis on $S$ in Theorem 5.11, the subtle point seems to be Claim 5.11.3. However, the above result and its proof already have some interesting consequences.

Remark 5.12. Since regular local rings are certainly quasi-Gorenstein, Theorem 5.11 immediately implies that Hochster-Huneke's vanishing conjecture for maps of Tor (or equivalently, the strong direct summand conjecture [Ran00]) implies the derived direct summand conjecture of Bhatt [Bha12].

Remark 5.13. The argument used in Theorem 5.11 gives a new proof that splinters and derived splinters are the same in characteristic $p>0$ for all local rings that are homomorphic image of Gorenstein local rings. First of all it is well known that splinters are CohenMacaulay in characteristic $p>0$ (we don't need completeness [HL07, [HH92]). Now the only place in the argument that we use the vanishing conditions for maps of Tor and the quasi-Gorenstein hypothesis seriously is in the proof of Claim 5.11.3. But this claim is clear in characteristic $p>0$ and we give a short argument as follows: by Theorem 2.1 of [HL07] we know that there exists a module-finite extension $B$ of $R$ such that the induced map $H_{\mathfrak{n}+R_{>0}}^{d}(R) \rightarrow H_{\mathfrak{n}+R_{>0}}^{d}(B)$ is zero. Since $B \otimes_{R} T$ is a module-finite extension of $T$ and hence a module-finite extension of $S$, the map $S \rightarrow B \otimes_{R} T$ splits as $S$-modules. Let

$$
\phi: T \rightarrow B \otimes_{R} T \stackrel{g}{\rightarrow} S
$$

be the composite map for some splitting $g: B \otimes_{R} T \rightarrow S$. We have the following commutative diagram:

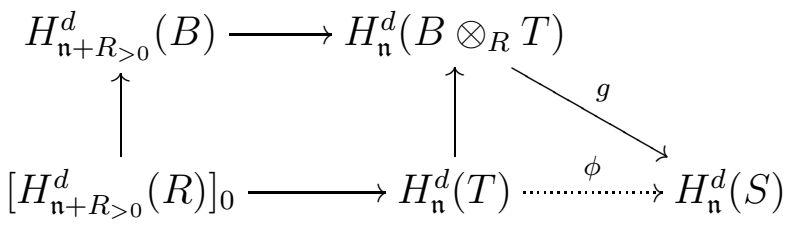

Since the left vertical map is the zero map by our choice of $B$, chasing through the diagram it is clear that the composite $\left[H_{\mathfrak{n}+R_{>0}}^{d}(R)\right]_{0} \rightarrow H_{\mathfrak{n}}^{d}(T) \stackrel{\phi}{\rightarrow} H_{\mathfrak{n}}^{d}(S)$ is the zero map. Hence Claim 
5.11.3 holds in characteristic $p>0$ as long as $S$ is a splinter (without any quasi-Gorenstein or complete hypothesis).

\section{ACKNOWLEDGEMENT}

It is a pleasure to thank Mel Hochster for several enjoyable discussions on the vanishing conjecture for maps of Tor and other homological conjectures. I want to thank Karl Schwede for reading a preliminary version of the paper and for his valuable comments. I would also like to thank Bhargav Bhatt and Anurag Singh for some helpful discussions. Finally I want to thank the referee, whose comments and suggestions lead to improvement of the paper.

\section{REFERENCES}

[Abe94] I. M. ABerbACH: Finite phantom projective dimension, Amer. J. Math. 116 (1994), no. 2, $447-477$.

[Bha12] B. BhAтт: Derived splinters in positive characteristic, Compos. Math. 148 (2012), no. 6, 17571786 .

[BST15] M. Blickle, K. Schwede, And K. TuCKer: F-singularities via alterations, Amer. J. Math 137 (2015), no. 1, 61-109.

[Bou87] J.-F. Boutot: Singularités rationnelles et quotients par les groupes réductifs, Invent. Math. 88 (1987), no. 1, 65-68.

[BE73] D. A. Buchsbau And D. Eisenbud: What makes a complex exact?, J. Algebra (1973), $259-268$.

[CEMS14] A. Chiecchio, F. Enescu, L. E. Miller, And K. Schwede: Test ideals in rings with finitely generated anti-canonical algebras, arXiv: 1412.6453.

[Eis95] D. EISENBUD: Commutative algebra with a view toward algebraic geometry, Springer-Verlag, New York, 1995.

[EV92] H. EsNault AND E. VIEHWEG: Lectures on vanishing theorems, DMV Seminar, vol. 20, Birkhäuser Verlag, Basel, 1992.

[GR70] H. GRAUERT AND O. RIEMEnschneIDER: Verschwindungssätze für analytische Kohomologiegruppen auf komplexen Räumen, Invent. Math. 11 (1970), 263-292.

[Har98] N. HARA: A characterization of rational singularities in terms of injectivity of Frobenius maps, Amer. J. Math. 120 (1998), no. 5, 981-996.

[Har66] R. HARTShorne: Residues and duality, Lecture notes of a seminar on the work of A. Grothendieck, given at Harvard 1963/64. With an appendix by P. Deligne. Lecture Notes in Mathematics, No. 20, Springer-Verlag, Berlin, 1966.

[Hei02] R. C. Heitmann: The direct summand conjecture in dimension three, Ann. of Math. (2) 156 (2002), no. 2, 695-712.

[Hoc02] M. Hochster: Big Cohen-Macaulay algebras in dimension three via Heitmann's theorem, J. Algebra 254 (2002), no. 2, 395-408.

[HH90] M. Hochster And C. Huneke: Tight closure, invariant theory, and the Briançon-Skoda theorem, J. Amer. Math. Soc. 3 (1990), no. 1, 31-116.

[HH92] M. Hochster And C. Huneke: Infinite integral extensions and big Cohen-Macaulay algebras, Ann. of Math. (2) 135 (1992), no. 1, 53-89.

[HH93] M. Hochster And C. Huneke: Phantom homology, Mem. Amer. Math. Soc. 103 (1993), no. 490 , vi+91.

[HH94] M. Hochster And C. Huneke: F-regularity, test elements, and smooth base change, Trans. Amer. Math. Soc. 346 (1994), no. 1, 1-62.

[HH95] M. Hochster And C. Huneke: Applications of the existence of big Cohen-Macaulay algebras, Adv. Math. 113 (1995), no. 1, 45-117.

[HR76] M. Hochster And J. L. Roberts: The purity of the Frobenius and local cohomology, Adv. Math. 21 (1976), no. 2, 117-172.

[HL07] C. Huneke And G. Lyubeznik: Absolute integral closure in positive characteristic, Adv. Math. 210 (2007), no. 2, 498-504. 
[HS03] E. Hyry AND K. E. Smith: On a non-vanishing conjecture of Kawamata and the core of an ideal, Amer. J. Math. 125 (2003), no. 6, 1349-1410.

[Kol86] J. KolLÁr: Higher direct images of dualizing sheaves I, Ann. of Math. (2) 123 (1986), no. 1, $11-42$.

[Kov00] S. Kovács: A characterization of rational singularities, Duke Math. J. 102 (2000), no. 2, 187191.

[Lip94] J. Lipman: Cohen-Macaulayness in graded algebras, Math. Res. Lett. 1 (1994), no. 2, 149-157.

[Lip02] J. Lipman: Lectures on local cohomology and duality, Local cohomology and its applications (Guanajuato, 1999), Lecture Notes in Pure and Appl. Math., vol. 226, Dekker, New York, 2002, pp. 39-89.

[LT81] J. Lipman And B. TeIssien: Pseudorational local rings and a theorem of Briançon-Skoda about integral closures of ideals, Michigan Math. J. 28 (1981), no. 1, 97-116.

[Ma14] L. MA: A sufficient condition for F-purity, J. Pure Appl. Algebra 218 (2014), 1179-1183.

[Ran00] N. Ranganathan: Splitting in module-finite extension rings and the vanishing conjecture for maps of Tor, Thesis, University of Michigan (2000).

[SdS87] J. B. SANCHO DE SAlas: Blowing-up morphisms with Cohen-Macaulay associated graded rings, Géométrie algébrique et applications, I (La Rábida, 1984), Travaux en Cours, vol. 22, Hermann, Paris, 1987, pp. 201-209.

[Sin99] A. K. SingH: Q-Gorenstein splinter rings of characteristic $p$ are F-regular, Math. Proc. Cambridge Philos. Soc. (1999), no. 2, 201-205.

[Smi94] K. E. Smith: Tight closure of parameter ideals, Invent. Math. 115 (1994), no. 1, 41-60.

[Smi97] K. E. Sмiтн: F-rational rings have rational singularities, Amer. J. Math. 119 (1997), no. 1, 159-180.

Department of Mathematics, University of Utah, Salt Lake City, Utah 84112

E-mail address: lquanma@math.utah.edu 